\title{
Generalized Thresholding and Online Sparsity-Aware Learning in a Union of Subspaces
}

\author{
Konstantinos Slavakis, \\ University of Minnesota, Digital Technology Center (DTC), \\ 487 Walter Library, 117 Pleasant St. SE, \\ Minneapolis, MN 55455, USA. \\ Email: slavakis@dtc.umn.edu
}

Yannis Kopsinis, University of Granada, Dept. of Applied Physics, Granada, Spain. ykopsinis@gmail.com

Sergios Theodoridis, University of Athens, Dept. of Informatics \& Telecommunications, Athens, Greece.

Email: stheodor@di.uoa.gr

Stephen McLaughlin, Heriot Watt University, School of Engineering \& Physical Sciences, Edinburgh, UK.

Email: S.McLaughlin@hw.ac.uk

\begin{abstract}
This paper studies a sparse signal recovery task in time-varying (time-adaptive) environments. The contribution of the paper to sparsity-aware online learning is threefold; first, a Generalized Thresholding (GT) operator, which relates to both convex and non-convex penalty functions, is introduced. This operator embodies, in a unified way, the majority of well-known thresholding rules which promote sparsity. Second, a non-convexly constrained, sparsity-promoting, online learning scheme, namely the Adaptive Projection-based Generalized Thresholding (APGT), is developed that incorporates the GT operator with a computational complexity that scales linearly to the number of unknowns. Third, the novel family of partially quasi-nonexpansive mappings is introduced as a functional analytic tool for treating the GT operator. By building upon the rich fixed point theory, the previous class of mappings helps us, also, to establish a link between the GT operator and a union of linear subspaces; a non-convex object which lies at the heart of any sparsity promoting technique, batch or online. Based on such a functional analytic framework, a convergence analysis of the APGT is provided. Furthermore, extensive experiments suggest that the APGT exhibits competitive performance when compared to computationally more demanding alternatives, such as the sparsity-promoting Affine Projection Algorithm (APA)- and Recursive Least Squares (RLS)based techniques.
\end{abstract}




\section{Introduction}

Sparsity-aware learning has been a topic at the forefront of research over the last ten years or so [1,2. Considerable effort has been invested in developing efficient schemes for the recovery of sparse signal/parameter vectors. However, most of these efforts have focussed on batch processing, via the Compressed Sensing or Sampling (CS) framework. In CS, an iterative algorithm is mobilized to solve the estimation task once all measurements (training data) have been collected by the processing unit [15]. It is only very recently that online (time-adaptive) algorithms have been developed, where the training data are processed sequentially, and the sparse signal to be recovered has the freedom to be time-varying [6-12]. Both CS and online techniques share a common strategy, namely thresholding; i.e, a thresholding rule is used to impose sparsity-aware a-priori knowledge: some of the components of the signal/vector to be estimated are kept intact, while the rest of them are shrunk under some user-defined rule. Two thresholding operators dominate the literature: (i) hard thresholding, a brute force method, where shrinking is achieved by setting the size of some of the vector components to zero, and (ii) soft thresholding, where the shrinking operation is based on the (weighted) $\ell_{1}$-norm of the vector.

A large number of thresholding operators have been studied thoroughly, both in theoretical and experimental contexts, mainly within the statistics community [13 27]. It is by now well-established that hard thresholding, a discontinuous operator, has a tendency for larger variance of the estimates. Moreover, due to its discontinuity, hard thresholding can lead to instabilities, in the sense of being sensitive to small changes in the training data [21. Soft-thresholding, is a continuous operator, that tends to introduce bias in the estimates. Therefore, alternative thresholding rules have been proposed in an effort to overcome these drawbacks [13, 18, 19, 24, 27]. These advances in thresholding operators are strongly connected to optimization tasks; they are obtained by minimizing squared error terms regularized by, usually, non-convex penalty functions.

The contribution of this paper is threefold. First, the generalized thresholding (GT) operator is introduced, which encompasses classical hard and soft thresholding rules, as well as the recent advances of [13 22, 24 27]. Moreover, the proposed framework, motivated by the rich fixed point theory [28, 29], is general enough to provide means for designing novel thresholding rules and/or incorporating a priori information associated with the sparsity level, i.e., the number of nonzero components, of the sparse vector to be recovered. More importantly, GT is also allowed to non-convexly constrain the unknown vector.

Second, the GT operator is incorporated into a signal/parameter estimation framework. Here, we choose the set theoretic estimation approach 30, and in particular its online version, introduced in 31 and extended in [32,33. In particular, the Adaptive Projection-based Generalized Thresholding (APGT) algorithm is proposed having three important merits. a) It is an online algorithm, b) it promotes sparse solutions effectively via the flexibility provided by the GT operator and c) its computational complexity scales linearly to the number of unknowns. With respect to performance, although APGT shows a low computational load, the experimental validation of Section 5 demonstrates that it exhibits a competitive performance even when compared to very recently developed, sparsity-promoting, and computationally more demanding alternatives, such as the APA- and RLS-based techniques [7, 34, 36.

It should be noted that the adopted set theoretic estimation framework was also utilized in [11, where sparsity was induced via $\ell_{1}$-based constraints, well-known to be convex and intimately connected to soft thresholding operations. In contrast, the fact that the GT operator is a "non-convex" mapping poses certain challenges for the convergence analysis of the algorithm. Specifically, the existing theory 31 33 which, so far, has been developed around convex sets and constraints is not rich enough to cover the APGT case. In order to theoretically support the incorporation of GT into learning mechanisms, such as the APGT, a novel family of operators, hereafter referred to as partially quasi-nonexpansive 
mappings, is introduced, to the best of our knowledge, for the first time. It is the introduction of the partially quasi-nonexpansive mappings and their nice properties, which allowed the convergence analysis of APGT to be developed. These operators serve as a sound theoretical tool which allows the use of variational analysis [37] and fixed point theory [28,29] to attack non-convexly constrained learning problems. It is shown that GT belongs to this class of nonlinear mappings, with its fixed point set being a union of subspaces; a non-convex object which lies at the heart of any sparsity-promoting technique [38, 39].

It should be stressed that, propelled by such a generic operator theoretical framework, the proposed GT mapping offers a sound mathematical basis for infusing sparsity arguments into both batch (CS) and online approaches, beyond the set-theoretic framework adopted here. Moreover, the present manuscript shows a value beyond sparsity-aware learning. Through the novel concept of the partially quasi-nonexpansive mappings, this study stands also as the first step toward the extension of [31 33 , to non-convexly constrained online learning tasks.

The remainder of the paper is organized as follows. The problem under consideration is stated in Section 2, In Section 3, the GT operator is introduced. The proposed APGT algorithm is given in Section 4, together with its properties and the definition of the novel family of partially quasi-nonexpansive mappings. Section 5 contains the experimental validation of APGT. A number of appendices support theoretically the developments exposed throughout the paper. More specifically, in App. C the properties of the generalized thresholding operator are studied rigorously, and the convergence analysis of the proposed algorithm is performed in App D, A preliminary version of this study was presented in [40].

\section{Problem Statement and Related Work}

We will denote the set of all non-negative integers, positive integers, and real numbers by $\mathbb{N}, \mathbb{N}_{*}$, and $\mathbb{R}$, respectively. Given any integers $j_{1}, j_{2}$, such that $j_{1} \leq j_{2}$, let $\overline{j_{1}, j_{2}}:=\left\{j_{1}, j_{1}+1, \ldots, j_{2}\right\}$.

The stage for discussion will be the Euclidean space $\mathbb{R}^{L}$, where $L \in \mathbb{N}_{*}$. Given any pair of vectors $\boldsymbol{a}_{1}, \boldsymbol{a}_{2} \in \mathbb{R}^{L}$, the inner product in $\mathbb{R}^{L}$ is defined as the classical vector-dot product $\left\langle\boldsymbol{a}_{1}, \boldsymbol{a}_{1}\right\rangle:=\boldsymbol{a}_{1}^{\top} \boldsymbol{a}_{2}$, where $T$ stands for vector/matrix transposition. The induced norm is $\|\cdot\|:=\sqrt{\langle\cdot, \cdot\rangle}$.

Our discussion will revolve around the following celebrated linear model:

$$
y_{n}=\boldsymbol{u}_{n}^{\top} \boldsymbol{a}_{*}+v_{n}, \quad \forall n \in \mathbb{N},
$$

where $\boldsymbol{a}_{*} \in \mathbb{R}^{L}$ is an unknown vector/signal, $\left(\boldsymbol{u}_{n}, y_{n}\right)_{n \in \mathbb{N}} \subset \mathbb{R}^{L} \times \mathbb{R}$ is a sequence of known training data, and $\left(v_{n}\right)_{n \in \mathbb{N}}$ stands for the noise process. In other words, the unknown $\boldsymbol{a}_{*}$ is "sensed" by a sequence of input vectors $\left(\boldsymbol{u}_{n}\right)_{n \in \mathbb{N}}$, via the inner product of $\mathbb{R}^{L}$, in order to produce the noisy outputs $\left(y_{n}\right)_{n \in \mathbb{N}}$. The vector $\boldsymbol{a}_{*}$ is considered to be sparse, i.e., most of its components are zero. If we define $\left\|\boldsymbol{a}_{*}\right\|_{0}$ to stand for the number of non-zero components of $\boldsymbol{a}_{*}$, then the assumption that $\boldsymbol{a}_{*}$ is sparse can be equivalently given by $K_{*}:=\left\|\boldsymbol{a}_{*}\right\|_{0} \ll L$, and the vector $\boldsymbol{a}_{*}$ will be called $K_{*}$-sparse.

This study attacks the following inverse problem: estimate the unknown sparse vector $\boldsymbol{a}_{*}$ by utilizing the sequence of training data $\left(\boldsymbol{u}_{n}, y_{n}\right)_{n \in \mathbb{N}}$. A family of algorithms which shares a similar objective is the Compressed Sensing or Sampling (CS) framework [1,2]. Given a fixed number $N \in \mathbb{N}_{*}$ of training data $\left(\boldsymbol{u}_{i}, y_{i}\right)_{i=n-N+1}^{n}$, a CS algorithm is mobilized in order to compute an estimate $\boldsymbol{a}_{n}$ of $\boldsymbol{a}_{*}$. CS belongs to the class of batch algorithms, i.e., in the case where the datum $\left(\boldsymbol{u}_{n+1}, y_{n+1}\right)$ enters the system, a CS algorithm starts from scratch, and triggers a generally time consuming iterative procedure which operates on the data $\left(\boldsymbol{u}_{i}, y_{i}\right)_{i=n-N+2}^{n+1}$ for computing the updated estimate $\boldsymbol{a}_{n+1}$ of $\boldsymbol{a}_{*}$. In contrast to batch learning approaches, this manuscript focuses on sparsity-aware online learning, i.e., an algorithmic framework which satisfies the following requirements. 
1. The estimates of $\boldsymbol{a}_{*}$ should be updated in a simple and efficient way every time that a new datum $\left(\boldsymbol{u}_{n}, y_{n}\right)$ enters the system. The need to mobilize an optimization procedure from scratch, for every new datum $\left(\boldsymbol{u}_{n}, y_{n}\right)$, as in CS, should be avoided.

2. The operations needed in order to update the estimate should be of low computational complexity; hopefully of linear complexity with respect to the number of unknowns, i.e., $\mathcal{O}(L)$.

3. The unknown $\boldsymbol{a}_{*}$ has also the freedom to be time-varying. Thus, an online learning scheme should be also able to quickly track any variations of $\boldsymbol{a}_{*}$.

The mainstream of sparsity-promoting online methods exploits training data $\left(\boldsymbol{u}_{n}, y_{n}\right)_{n \in \mathbb{N}}$ in the context of classical adaptive filtering [41; a quadratic objective function is used to quantify the designer's perception of loss. Additionally, a convex differentiable function is regularized by a sparsity promoting term, usually one that builds around the $\ell_{1}$ norm penalty function, and a minimizer of the resulting optimization task is sought either in the RLS or the LMS rationale, e.g., 66 9]. Another sparsitypromoting methodology, where different components of the vector estimates are weighted under several user-defined rules, is given by proportionate-type schemes [34 36. Very recently, a novel online method for the recovery of sparse signals, based on set theoretic estimation arguments [30,42, was developed in [11, and extended for distributed learning in [43].

The set theoretic estimation philosophy departs from the standard approach of constructing a loss function first; instead, it initially identifies a set of solutions which are in agreement with the available measurements as well as the available a-priori knowledge. A popular strategy is to define, at each time instance $n \in \mathbb{N}$, a closed convex subset of $\mathbb{R}^{L}$, by means of the training data pair $\left(\boldsymbol{u}_{n}, y_{n}\right)$, to contain the unknown $\boldsymbol{a}_{*}$ with high probability. Different alternatives exist on how to "construct" such convex regions. A popular choice takes the form of a hyperslab around $\left(\boldsymbol{u}_{n}, y_{n}\right)$, which is defined as:

$$
S_{n}\left[\epsilon_{n}\right]:=\left\{\boldsymbol{a} \in \mathbb{R}^{L}:\left|\boldsymbol{u}_{n}^{\top} \boldsymbol{a}-y_{n}\right| \leq \epsilon_{n}\right\}, \quad \forall n \in \mathbb{N},
$$

for some user-defined tolerance $\epsilon_{n} \geq 0$, and for $\boldsymbol{u}_{n} \neq \mathbf{0}$. The parameter $\epsilon_{n}$ determines, essentially, the width of the hyperslabs, and it implicitly models the effects of the noise, as well as various other uncertainties, like measurement inaccuracies, calibration errors, etc. For example, if the noise were bounded, i.e., $\exists \rho \geq 0$ such that $\left|v_{n}\right| \leq \rho, \forall n \in \mathbb{N}$, then for any choice of $\epsilon_{n} \geq \rho$ it is easy to verify that $\boldsymbol{a}_{*} \in S_{n}\left[\epsilon_{n}\right], \forall n \in \mathbb{N}$. A rigorous stochastic analysis in the case of bounded noise, where almost sure convergence of the sequence of estimates is proved for a special member of the rich family of the Adaptive Projected Subgradient Method (APSM) [31 33], can be found in [44. In the case of unbounded noise, the well-known Tchebichev inequality [45] suggests that for any $\epsilon_{n}>0$,

$$
\operatorname{Prob}\left\{\boldsymbol{a}_{*} \in S_{n}\left[\epsilon_{n}\right]\right\}=\operatorname{Prob}\left\{\left|\boldsymbol{u}_{n}^{\top} \boldsymbol{a}_{*}-y_{n}\right| \leq \epsilon_{n}\right\} \geq 1-\frac{\mathrm{E}\left\{\left|\boldsymbol{u}_{n}^{\top} \boldsymbol{a}_{*}-y_{n}\right|^{2}\right\}}{\epsilon_{n}^{2}}=1-\frac{\mathrm{E}\left\{\left|v_{n}\right|^{2}\right\}}{\epsilon_{n}^{2}},
$$

where Prob denotes probability, and E stands for the expectation operator. In other words, $\epsilon_{n}$ defines also a measure of confidence in having the unknown $\boldsymbol{a}_{*}$ in the hyperslabs (2).

The (metric) projection mapping $P_{S_{n}\left[\epsilon_{n}\right]}$ [29] onto the hyperslab $S_{n}\left[\epsilon_{n}\right]$ (2) is given by the following simple analytic formula:

$$
P_{S_{n}\left[\epsilon_{n}\right]}(\boldsymbol{a})=\boldsymbol{a}+ \begin{cases}\frac{y_{n}-\epsilon_{n}-\boldsymbol{u}_{n}^{\top} \boldsymbol{a}}{\left\|\boldsymbol{u}_{n}\right\|^{2}} \boldsymbol{u}_{n}, & \text { if } y_{n}-\epsilon_{n}>\boldsymbol{u}_{n}^{\top} \boldsymbol{a}, \\ 0, & \text { if }\left|\boldsymbol{u}_{n}^{\top} \boldsymbol{a}-y_{n}\right| \leq \epsilon_{n}, \\ \frac{y_{n}+\epsilon_{n}-\boldsymbol{u}_{n}^{\top} \boldsymbol{a}}{\left\|\boldsymbol{u}_{n}\right\|^{2}} \boldsymbol{u}_{n}, & \text { if } y_{n}+\epsilon_{n}<\boldsymbol{u}_{n}^{\top} \boldsymbol{a} .\end{cases}
$$


In [11] sparsity was induced within the convex analytic framework, and particularly via projections onto convex $\ell_{1}$-balls. Here, the fixed point theoretical framework [28, 29] is used in order to generalize the set theoretic estimation approach to support sparsity promoting constraints, which do not lie under the umbrella of convexity. This is realized via a novel operator theoretic framework, which embraces a wide range of thresholding rules referred to as Generalized Thresholding (GT) operators, described next.

\section{The Generalized Thresholding (GT) Mapping}

A couple of definitions are necessary prior to introducing GT mapping.

Definition 1 (The ordered tuple notation). Given $K \in \overline{1, L}$, define the set of all ascending tuples of length $K$ as $\mathscr{T}(K, L):=\left\{\left(l_{1}, l_{2}, \ldots, l_{K}\right): 1 \leq l_{1}<l_{2}<\ldots<l_{K} \leq L\right\}$. Clearly, the cardinality of $\mathscr{T}(K, L)$ is $\left(\begin{array}{c}L \\ K\end{array}\right)$. An example of such an ordered tuple is the support of a vector $\boldsymbol{x} \in \mathbb{R}^{L}$, defined by $\operatorname{supp}(\boldsymbol{x}):=\left(l \in \overline{1, L}: x_{l} \neq 0\right) \in \mathscr{T}(|\operatorname{supp}(\boldsymbol{x})|, L)$, where $|\cdot|$ stands for the cardinality of a set.

Definition 2 (Subspace associated to a tuple). Given $J \in \mathscr{T}(K, L)$, let $M_{J}:=\left\{\boldsymbol{a} \in \mathbb{R}^{L}: a_{l}=0, \forall l \notin J\right\}$. Clearly, $M_{J}$ is a linear subspace of $\mathbb{R}^{L}$. Moreover, notice that if $J_{1} \subset J_{2}$, then $M_{J_{1}} \subset M_{J_{2}}$. In particular, if $\operatorname{supp}\left(\boldsymbol{x}_{*}\right) \subset J$, then $\boldsymbol{x}_{*} \in M_{J}$. An illustration of $M_{J}$ can be found in Fig. 1.

Motivated by the hard thresholding operator, let us introduce here the main object of this study.

Definition 3 (The mapping $T_{\mathrm{GT}}^{(K)}$ ). Fix a positive integer $K \in \overline{1, L-1}$ and define $T_{\mathrm{GT}}^{(K)}: \mathbb{R}^{L} \rightarrow \mathbb{R}^{L}$ as follows. For any $\boldsymbol{x} \in \mathbb{R}^{L}$, the output $\boldsymbol{z}:=T_{\mathrm{GT}}^{(K)}(\boldsymbol{x})$ is obtained according to the following steps:

1. Compute, first, the tuple $J_{\boldsymbol{x}}^{(K)} \in \mathscr{T}(K, L)$ which contains the indices of the $K$ largest, in absolute value, components of $\boldsymbol{x}$. To avoid any ambiguity, in the case where we identify more than one component of $\boldsymbol{x}$ with the same absolute value, we always choose the one with smallest index.

2. Define $\xi_{\boldsymbol{x}}^{(K)}:=\min \left\{\left|x_{l}\right|: l \in J_{\boldsymbol{x}}^{(K)}\right\}$. In words, $\xi_{\boldsymbol{x}}^{(K)}$ is the smallest among the $K$ largest absolute values of the components of $\boldsymbol{x}$. Clearly, $\forall l \notin J_{\boldsymbol{x}}^{(K)},\left|x_{l}\right| \leq \xi_{\boldsymbol{x}}^{(K)}$.

3. Compute the components of $\boldsymbol{z}$ as: $z_{l}:=x_{l}$, if $l \in J_{\boldsymbol{x}}^{(K)}$, and $z_{l}:=\operatorname{shr}\left(x_{l}\right)$, if $l \notin J_{\boldsymbol{x}}^{(K)}$, where the function shr: $\mathcal{D}_{\boldsymbol{x}} \rightarrow \mathbb{R}$, with $\mathcal{D}_{\boldsymbol{x}}:=\left[-\xi_{\boldsymbol{x}}^{(K)}, \xi_{\boldsymbol{x}}^{(K)}\right]$, satisfies the following properties:

4. $\tau \operatorname{shr}(\tau) \geq 0, \forall \tau \in \mathcal{D}_{\boldsymbol{x}}$.

5. $|\operatorname{shr}(\tau)| \leq|\tau|, \forall \tau \in \mathcal{D}_{\boldsymbol{x}}$

6. Going a step further than the previous property, we assume also that given any sufficiently small $\epsilon>0$, there exists a $\delta>0$, such that for any $\boldsymbol{x} \in \mathbb{R}^{L}$, and $\forall \tau \in \mathcal{D}_{\boldsymbol{x}} \backslash(-\epsilon, \epsilon),|\operatorname{shr}(\tau)| \leq|\tau|-\delta$. In other words, $\delta$ could be a user-defined parameter which guarantees that the function shr acts as a strict shrinkage operator for all the components of $\boldsymbol{x}$ with indexes not in $J_{\boldsymbol{x}}^{(K)}$. The $\epsilon$ parameter is introduced in order to exclude 0 from the picture, since at this point the shr function usually takes the value of 0 , i.e., $\operatorname{shr}(0)=0$ (see Fig. 21).

Put in other words, the GT mapping operates as follows; given the input vector $\boldsymbol{x}$, a number of $K$ components of $\boldsymbol{x}$, i.e., those with the $K$ largest absolute values, are kept intact, while the rest of them are shrunk according to the shr function. See, for example, Fig. 1, 


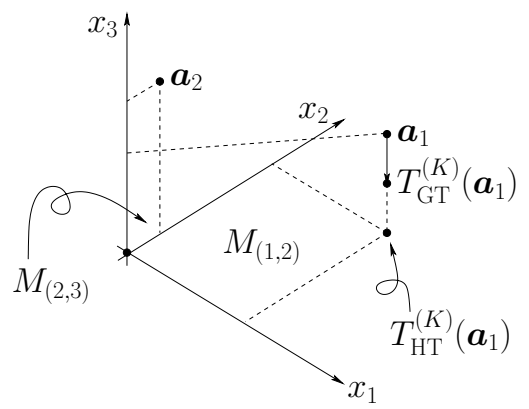

Figure 1: An illustration of $T_{\mathrm{GT}}^{(K)}$, for the case of a 3 -dimensional space, i.e., $L:=3$, and $K:=2$. Take for example the point $\boldsymbol{a}_{1}$. The $K=2$ largest, in magnitude, coordinates of $\boldsymbol{a}_{1}$ are the first two ones, i.e., $J_{\boldsymbol{a}_{1}}^{(K)}=(1,2)$. The linear subspace $M_{(1,2)}$ stands for all those vectors in $\mathbb{R}^{3}$ where all the components, except from those in the positions $(1,2)$, are equal to 0 . The first two components of $\boldsymbol{a}_{1}$ stay unaffected by $T_{\mathrm{GT}}^{(K)}$, while the third one is shrinked by the function shr. If this third coordinate is set to 0 , then $T_{\mathrm{GT}}^{(K)}$ acts as the hard-thresholding mapping $T_{\mathrm{HT}}^{(K)}$. On the other hand, the point $\boldsymbol{a}_{2}$ is already located in $M_{(2,3)}$, i.e., its first coordinate is 0 . Hence, the application of $T_{\mathrm{GT}}^{(K)}$ to $\boldsymbol{a}_{2}$ has no effect, and $\boldsymbol{a}_{2}$ stays fixed to its original position.
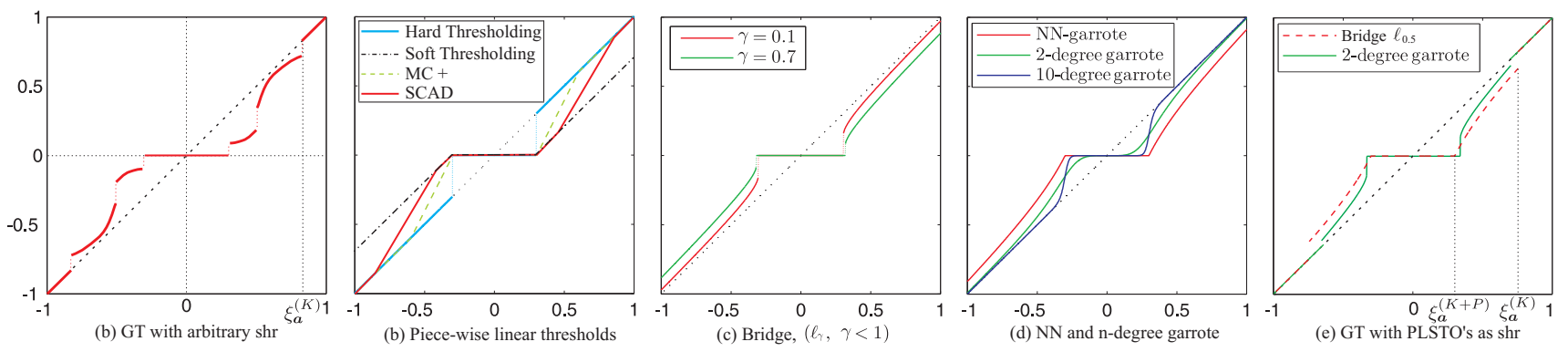

Figure 2: Plots of Penalized Least-Squares Thresholding Operators (PLSTO) for various choices of the penalty function $p$ in (5) .

The function shr is user-defined and it can get various forms as long as it complies with the properties described before. As an example, a thresholding operator in the GT family based on an arbitrary shr function is shown in Fig. 2a. Note that it comprises both discontinuities and nonlinear regions. A more systematic way to built GT's is via the univariate Penalized Least Squares optimization task; given $\tilde{a} \in \mathbb{R}$

$$
\min _{a} \frac{1}{2}(\tilde{a}-a)^{2}+\lambda p(|a|)
$$

where $p(\cdot)$ is nonnegative, nondecreasing and differentiable function on $(0, \infty)$. This problem is at the heart of many batch sparsity promoting algorithms as it is discussed in App. B. It turns out, that (44) has, in general, a unique solution which is obtained when $\tilde{a}$ is properly thresholded/shrinked [21]. Accordingly, let us define the Penalized Least-Squares Thresholding Operator (PLSTO) as the mapping which maps a given $\tilde{a}$ to the previous unique minimizer:

$$
T_{\mathrm{PLSTO}}^{(p, \lambda)}: \tilde{a} \mapsto \arg \min _{a \in \mathbb{R}} \frac{1}{2 \lambda}(\tilde{a}-a)^{2}+p(|a|) .
$$

In simple words, the PLSTO of (51) shrinks, in some sense that is dictated by $p$, the size of $\tilde{a}$. Examples 
of PLSTO's exhibiting different characteristics are shown in Fig. 2(b-d) and details together with the corresponding literature review can be found in App. B. All the thresholding rules of Fig. 2(b-d) satisfy the properties of Def. 314 and Def. 315. Moreover, they also satisfy the property of Def. 36] in their respective strict-shrinkage region, i.e., in the case where the $\xi_{\boldsymbol{x}}^{(K)}$ lies in the domain of all those $\tau \in \mathbb{R}$ such that $\left|T_{\mathrm{PLSTO}}^{(p)}(\tau)\right|<|\tau|$. Notice, also, that we do not impose any regularity conditions on shr, like continuity or differentiability, unlike most of the known PLSTO do [13, 19, 24, 27]. As a result, any PLSTO, i.e., (5), can be used in the place of the shr function in the GT operator. Examples of GT having PLSTO's as their shr function are shown in Figs. 2a and 20. Moreover, GT where the shr function is the Bridge $\ell_{0.5}$ and the Smoothly Clipped Absolute Deviation Penalty (SCAD) threshold are used and further discussed in the numerical experiments section.

\section{The APGT Algorithm, Its Properties, and a Novel Operator The- oretic Framework}

Algorithm 1 (The Adaptive Projection-based Generalized Thresholding (APGT) algorithm). Given the user-defined sparsity level $K \in \overline{1, L-1}$, the sequence of non-negative parameters $\left(\epsilon_{n}\right)_{n \in \mathbb{N}}$, the number $q \in \mathbb{N}_{*}$ of the hyperslabs to be processed concurrently at every time instant, the function shr for the generalized thresholding operation, and an arbitrary initial point, $\boldsymbol{a}_{0} \in \mathbb{R}^{L}$, execute the following, for every $n \in \mathbb{N}$.

1. Define the sliding window $\mathcal{J}_{n}:=\overline{\max \{0, n-q+1\}, n}$ on the time axis, of size at most $q$. The set $\mathcal{J}_{n}$ defines all the indices corresponding to the hyperslabs, which are to be processed at the time instant $n$. Among these, identify $\mathcal{I}_{n}:=\left\{i \in \mathcal{J}_{n}: P_{S_{i}\left[\epsilon_{i}\right]}\left(\boldsymbol{a}_{n}\right) \neq \boldsymbol{a}_{n}\right\}$, which correspond to the active hyperslabs. Moreover, for every $i \in \mathcal{I}_{n}$, define the weight $\omega_{i}^{(n)}:=1 /\left|\mathcal{I}_{n}\right|$, where $\left|\mathcal{I}_{n}\right|$ denotes the cardinality of $\mathcal{I}_{n}$, in order to weigh uniformly the importance of the information carried by each hyperslab, $S_{i}\left[\epsilon_{i}\right]$. Other, more general, scenarios regarding the choice of $\left\{\omega_{i}^{(n)}\right\}_{i=1}^{\left|\mathcal{I}_{n}\right|}$ are also possible.

2. Collect the projections $P_{S_{i}\left[\epsilon_{i}\right]}\left(\boldsymbol{a}_{n}\right), \forall i \in \mathcal{I}_{n}$ (see (3) ).

3. Choose an $\varepsilon^{\prime} \in(0,1]$, and let the extrapolation parameter $\mu_{n}$ take values from the interval $\left[\varepsilon^{\prime} \mathcal{M}_{n},(2-\right.$ $\left.\left.\varepsilon^{\prime}\right) \mathcal{M}_{n}\right]$, where

$$
\mathcal{M}_{n}:=\left\{\begin{array}{l}
\frac{\sum_{i \in \mathcal{I}_{n}} \omega_{i}^{(n)}\left\|P_{S_{i}\left[\epsilon_{i}\right]}\left(\boldsymbol{a}_{n}\right)-\boldsymbol{a}_{n}\right\|^{2}}{\left\|\sum_{i \in \mathcal{I}_{n}} \omega_{i}^{(n)} P_{S_{i}\left[\epsilon_{i}\right]}\left(\boldsymbol{a}_{n}\right)-\boldsymbol{a}_{n}\right\|^{2}} \\
\quad \text { if } \sum_{i \in \mathcal{I}_{n}} \omega_{i}^{(n)} P_{S_{i}\left[\epsilon_{i}\right]}\left(\boldsymbol{a}_{n}\right) \neq \boldsymbol{a}_{n} \\
\quad \text { otherwise. }
\end{array}\right.
$$

Notice that due to the convexity of the function $\|\cdot\|^{2}$, we always have $\mathcal{M}_{n} \geq 1$. As such, the parameter $\mu_{n}$ takes values larger than or equal to 2 . In general, the larger the $\mu_{n}$, the larger the convergence speed of the proposed algorithm.

4. Compute the next estimate by

$$
\boldsymbol{a}_{n+1}:= \begin{cases}T_{\mathrm{GT}}^{(K)}\left(\boldsymbol{a}_{n}+\mu_{n}\left(\sum_{i \in \mathcal{I}_{n}} \omega_{i}^{(n)} P_{S_{i}\left[\epsilon_{i}\right]}\left(\boldsymbol{a}_{n}\right)-\boldsymbol{a}_{n}\right)\right), \\ T_{\mathrm{GT}}^{(K)}\left(\boldsymbol{a}_{n}\right), & \text { if } \mathcal{I}_{n} \neq \emptyset, \\ \text { if } \mathcal{I}_{n}=\emptyset .\end{cases}
$$


In order to theoretically support the incorporation of GT into parameter estimation schemes, a novel family of mappings, called the partially quasi-nonexpansive mappings, which, to the best of our knowledge, appears for the first time in the related literature [29]. The reasons for defining this new class of mappings are: (i) this family includes as a special case the previously defined generalized thresholding operator $T_{\mathrm{GT}}^{(K)}$, and, thus, it establishes a general theoretical framework for sparsity-promoting mappings, (ii) it introduces sound theoretical tools, which help to attack non-convexly constrained learning problems, and (iii) it generalizes the very recent results, obtained for the Adaptive Projected Subgradient Method (APSM) [33, to non-convexly constrained online learning tasks (see App. D).

Although the following discussion can be naturally extended to general Hilbert spaces, for the sake of simplicity we focus here on the Euclidean space $\mathbb{R}^{L}$, i.e., $T: \mathbb{R}^{L} \rightarrow \mathbb{R}^{L}$. A concept of fundamental importance, associated to every mapping $T$, is its fixed point set $\operatorname{Fix}(T):=\left\{\boldsymbol{a} \in \mathbb{R}^{L}: T(\boldsymbol{a})=\boldsymbol{a}\right\}$ [28, 29]. In other words, $\operatorname{Fix}(T)$ reveals the hidden modes of $T$, by putting together all those points unaffected by $T$. To leave no place for ambiguity, every $\operatorname{Fix}(T)$ that appears in the sequel is assumed nonempty.

Definition 4 (The class of partially quasi-nonexpansive mappings). A mapping $T$ is called partially quasi-nonexpansive, if

$$
\begin{gathered}
\forall \boldsymbol{x} \in \mathbb{R}^{L}, \exists Y_{\boldsymbol{x}} \subset \operatorname{Fix}(T): \forall \boldsymbol{y} \in Y_{\boldsymbol{x}}, \\
\|T(\boldsymbol{x})-\boldsymbol{y}\| \leq\|\boldsymbol{x}-\boldsymbol{y}\| .
\end{gathered}
$$

The fixed point set $\operatorname{Fix}(T)$ is not necessarily a convex set. Let us also define a stronger version of (7); the mapping $T$ will be called strongly or $\eta$-attracting partially quasi-nonexpansive mapping if there exists an $\eta>0$ such that

$$
\begin{gathered}
\forall \boldsymbol{x} \in \mathbb{R}^{L}, \exists Y_{\boldsymbol{x}} \subset \operatorname{Fix}(T): \forall \boldsymbol{y} \in Y_{\boldsymbol{x}}, \\
\eta\|\boldsymbol{x}-T(\boldsymbol{x})\|^{2} \leq\|\boldsymbol{x}-\boldsymbol{y}\|^{2}-\|T(\boldsymbol{x})-\boldsymbol{y}\|^{2} .
\end{gathered}
$$

An example of such a mapping (8) is the novel generalized thresholding mapping of Section 3 (for a proof see App.Cl). In App. C, we will also verify that Fix $\left(T_{\mathrm{GT}}^{(K)}\right)$ is a union of subspaces, which is indeed a non-convex set. Recall that at the heart of any sparsity-promoting learning method lies the search for a solution in a union of subspaces [38,39]. It must be pointed out that a number of well-known mappings, e.g., [10, 29, 46], are special cases of the previously defined class of partially quasi-nonexpansive ones.

The convergence analysis of the APGT is given by the following Thm. 1. This analysis is based on a set of deterministic assumptions, given below. Since the APGT is based on the mapping $T_{\mathrm{GT}}^{(K)}$, whose fixed point set (see App. C) is non-convex, this is the first time that the results of [31 33] are generalized to non-convexly constrained online learning tasks.

\section{Assumption 1.}

1. Assume that $\exists n \in \mathbb{N}$ such that $\Omega_{n}:=M_{J_{a_{n}}^{(K)}} \cap \bigcap_{i \in \mathcal{I}_{n}} S_{i}\left[\epsilon_{i}\right] \neq \emptyset$. Let us explain here the physical reasoning behind this assumption. Recall, here, that $\left\{S_{i}\left[\epsilon_{i}\right]\right\}_{i \in \mathcal{I}_{n}}$ is the set of all active hyperslabs (see Alg. 1), at the time instant $n$. For an appropriate choice of the parameters $\left(\epsilon_{n}\right)_{n \in \mathbb{N}}($ see (2) ), the hyperslabs contain the desired $\boldsymbol{a}_{*}$ with high probability. Moreover, as time goes by, and due to a long sequence of projections in (6), the orbit $\left(\boldsymbol{a}_{n}\right)_{n \in \mathbb{N}}$ is attracted closer and closer to the hyperslabs; and as a consequence, closer to $\boldsymbol{a}_{*}$. For this reason, it is natural to expect that $\operatorname{supp}\left(\boldsymbol{a}_{n}\right)$ is $\operatorname{similar}$ to $\operatorname{supp}\left(\boldsymbol{a}_{*}\right)$, and hence $M_{J_{\boldsymbol{a}_{n}}^{(K)}}$ to $M_{J_{\boldsymbol{a}_{*}}^{(K)}}$, at some time $n$. Since $M_{J_{\boldsymbol{a}_{*}}^{(K)}}$ enjoys a non-empty intersection with $\bigcap_{i \in \mathcal{I}_{n}} S_{i}\left[\epsilon_{i}\right]$, with high probability, we anticipate that the same also happens to $M_{J_{a_{n}}^{(K)}}$.

2. Assume that there exists a time instant $n_{0} \in \mathbb{N}$, and an $N \in \mathbb{N}_{*}$, such that $\bigcap_{n=n_{0}}^{n_{0}+N-1} \Omega_{n} \neq \emptyset$. 
3. Assume that $\Omega:=\liminf _{n \rightarrow \infty} \Omega_{n}:=\bigcup_{n \geq 0} \bigcap_{m \geq n} \Omega_{m} \neq \emptyset$. In other words, we assume that the set of all points, which belong to all but a finite number of $\Omega_{n}$ s, is nonempty.

Theorem 1 (Properties of the APGT).

1. Let Assumption 11 hold true. Then, $d\left(\boldsymbol{a}_{n+1}, \Omega_{n}\right) \leq d\left(\boldsymbol{a}_{n}, \Omega_{n}\right)$, where $d\left(\cdot, \Omega_{n}\right)$ stands for the (metric) distance function [29] to $\Omega_{n}$.

2. Let Assumption 112 hold true. Then,

$$
\begin{aligned}
d^{2}\left(\boldsymbol{a}_{n_{0}+N},\right. & \left.\bigcap_{n=n_{0}}^{n_{0}+N-1} \Omega_{n}\right) \leq d^{2}\left(\boldsymbol{a}_{n_{0}}, \bigcap_{n=n_{0}}^{n_{0}+N-1} \Omega_{n}\right) \\
& -\frac{\left(\varepsilon^{\prime}\right)^{2}}{q} \sum_{n=n_{0}}^{n_{0}+N-1} \max \left\{d^{2}\left(\boldsymbol{a}_{n}, S_{j}\left[\epsilon_{j}\right]\right): j \in \mathcal{J}_{n}\right\} .
\end{aligned}
$$

In other words, the previous inequality establishes a bound on the distance of the estimates from a finite intersection of the $\Omega_{n}$ s. If we assume, also, that there exists an estimate $\boldsymbol{a}_{n}$ which does not belong to such an intersection, i.e., $\exists n^{\prime} \in \overline{n_{0}, n_{0}+N-1}$ such that $\max \left\{d^{2}\left(\boldsymbol{a}_{n^{\prime}}, S_{j}\left[\epsilon_{j}\right]\right): j \in \mathcal{J}_{n^{\prime}}\right\}>0$, then the previous result claims that the APGT forces $\boldsymbol{a}_{n_{0}+N}$ to be located strictly closer to $\bigcap_{n=n_{0}}^{n_{0}+N-1} \Omega_{n}$ than $\boldsymbol{a}_{n_{0}}$ is.

3. Let Assumption 113 holds true. Then,

(a) the set of all cluster points of the sequence $\left(\boldsymbol{a}_{n}\right)_{n \in \mathbb{N}}$ is nonempty, i.e., $\mathfrak{C}\left(\left(\boldsymbol{a}_{n}\right)_{n \in \mathbb{N}}\right) \neq \emptyset$.

(b) $\lim _{n \rightarrow \infty} d\left(\boldsymbol{a}_{n}, S_{n}\left[\epsilon_{n}\right]\right)=0$. In other words, as the time advances, the orbit $\left(\boldsymbol{a}_{n}\right)_{n \in \mathbb{N}}$ approaches $\left(S_{n}\left[\epsilon_{n}\right]\right)_{n \in \mathbb{N}}$.

(c) $\mathfrak{C}\left(\left(\boldsymbol{a}_{n}\right)_{n \in \mathbb{N}}\right) \subset \operatorname{Fix}\left(T_{\mathrm{GT}}^{(K)}\right)=\bigcup_{J \in \mathscr{T}(K, L)} M_{J}$. In words, the APGT generates a sequence of estimates $\left(\boldsymbol{a}_{n}\right)_{n \in \mathbb{N}}$, whose cluster points are sparse vectors, of sparsity level no larger than $K$.

Proof. See Appendix D.

\section{$5 \quad$ Numerical Experiments}

In this section, our main intention is to provide the proof of concept of the theoretical findings presented in Thm. 1. This is realized via the performance evaluation of (6b), where the shrinkage function shr, in Def. 3, assumes any form of $T_{\mathrm{PLSTO}}^{(p)}$, defined in (5). This study is not meant to be exhaustive, and in order to demonstrate the potential of the proposed technique, the hard thresholding (HT) as well as the PLSTOs corresponding to the SCAD [19] and the $\ell_{\gamma}$ penalty $(\gamma<1)[13$ are examined, since they exhibit distinct characteristics, as it is illustrated in Figs. $2 \mathrm{~b}$ and 2 $\mathrm{k}$, respectively. Notice that the associated penalty functions are non-convex. The resulting thresholding rules are called the $S C A D$ and the Bridge Thresholding (BT), respectively. Notice, also, that SCAD is a piece-wise linear thresholding operator, whereas, the BT exhibits strong discontinuity and non-linearity.

In order to comply with the theory, the SCAD, the BT, and the HT are used as shrinkage functions shr in Def. 3, for all the components $x_{i}$ with index $i \notin J_{x}^{(K)}$, where $K$ stands for an estimate of the true $K_{*}:=\left\|\boldsymbol{a}_{*}\right\|_{0}$. To this end, we have slightly modified the classical SCAD, BT, and HT rules in order to fit our need to keep a number of $K$ components of a vector intact. As such, the SCAD thresholding 
operates according to the following rule; given the input $\boldsymbol{x} \in \mathbb{R}^{L}$ and the output vector $\boldsymbol{z}:=T_{\mathrm{SCAD}}(\boldsymbol{x})$, the $i$-th coordinate of $\boldsymbol{z}$, where $i \notin J_{\boldsymbol{x}}^{(K)}$, is given by the next rule:

$$
z_{i}= \begin{cases}0, & \text { if }\left|x_{i}\right| \leq \lambda, \\ \operatorname{sgn}\left(x_{i}\right)\left(\left|x_{i}\right|-\lambda-\delta\right)_{+}, & \text {if }\left|x_{i}\right| \in(\lambda, 2 \lambda], \\ \operatorname{sgn}\left(x_{i}\right)\left(\frac{(\alpha-1)\left|x_{i}\right|-\alpha \lambda}{\alpha-2}-\delta\right)_{+}, & \\ \quad \text { if }\left|x_{i}\right| \in\left(2 \lambda, \min \left\{\xi_{x}^{(K)}, \alpha \lambda\right\}\right],\end{cases}
$$

where $\lambda$ is the regularization parameter, which appears in the definition of the PLSTO in (5), $\alpha$ is a userdefined parameter, inherent to SCAD [19], $\delta>0$ is a sufficiently small user-defined parameter motivated by Definition 36, and $(\cdot)_{+}:=\max \{0, \cdot\}$, introduced here in order to leave no place for ambiguities. Our modification on the classical SCAD can be seen by the introduction of $\delta, \xi_{x}^{(K)}$, and $(\cdot)_{+}$.

Similarly, given the classical version of the BT rule [18], our modified BT is given as follows by involving the quantity $\xi_{\boldsymbol{x}}^{(K)}$ in the computations: $\forall i \notin J_{\boldsymbol{x}}^{(K)}$,

$$
z_{i}=\left\{\begin{aligned}
\operatorname{sgn}\left(x_{i}\right)\left(\bar{z}_{i}-\delta\right)_{+} & \\
\quad & \text { if } \min \left\{c_{\mathrm{BT}}(\lambda, \gamma), \xi_{\boldsymbol{x}}^{(K)}\right\} \leq\left|x_{i}\right| \leq \xi_{\boldsymbol{x}}^{(K)} \\
0, & \text { otherwise }
\end{aligned}\right.
$$

where $\lambda$ is the corresponding regularization parameter in (5), $\gamma \in(0,1)$ is a user-defined parameter, and

$$
c_{\mathrm{BT}}(\lambda, \gamma):=\left(-\frac{1}{\lambda \gamma(\gamma-1)}\right)^{\frac{1}{\gamma-2}}+\lambda \gamma\left(-\frac{1}{\lambda \gamma(\gamma-1)}\right)^{\frac{\gamma-1}{\gamma-2}} .
$$

The term $\bar{z}_{i}$ stands for the solution of the equation $\bar{z}_{i}+\operatorname{sgn}\left(z_{i}\right) \lambda \gamma \bar{z}_{i}^{\gamma-1}=\left|x_{i}\right|$. When $\gamma$ is set equal to $0.5, \bar{z}_{i}$ is obtained in closed form by solving a third order polynomial equation. Similarly, HT is given by the following rule; $\forall i \notin J_{\boldsymbol{x}}^{(K)}$,

$$
z_{i}= \begin{cases}0, & \text { if }\left|x_{i}\right| \leq \min \left\{\lambda, \xi_{\boldsymbol{x}}^{(K)}\right\}, \\ \operatorname{sgn}\left(x_{i}\right)\left(\left|x_{i}\right|-\delta\right)_{+}, & \text {otherwise, }\end{cases}
$$

where the $\lambda$ is introduced here in order to be compliant also to a definition of the HT used often in the literature (see the discussion in Appendix B).

In the following experiments, unless otherwise stated, the signal under consideration has $L=1024$ and $K_{*}=100$. Moreover, the classical CS signal recovery problem is considered, where the input (sensing) vectors have independent components drawn from a normal distribution $\mathcal{N}(0,1)$, and the observations are corrupted by additive white Gaussian noise of variance $\sigma^{2}=0.1$. Regarding APGT, the extrapolation parameter $\mu_{n}$ is set equal to $\mathcal{M}_{n}$, and the hyperslab parameter $\epsilon_{n}:=1.3 \sigma, \forall n$. In this paper, for all the techniques employed, configurations leading to the fastest convergence rate are of principal interest. From this perspective, unless otherwise stated, $q$ is fixed to 390 since this appeared to be the lowest $q$ value leading to enhanced convergence speed for the specific $L$ and $K$ values. It should be stressed out that the method is not sensitive to the parameter $q$. A larger $q$ value would only add to computational complexity without any significant contribution to performance. An extensive and complementary experimental study of the APGT performance, in the case where $q$ is confined to small values, which relates to very low computational complexity techniques, can be found in [47, 48]. In all of the succeeding figures, the MSE stands for $\mathrm{MSE}_{n}:=\frac{1}{\tau L} \sum_{i=1}^{\tau}\left\|\boldsymbol{a}_{*}-\boldsymbol{a}_{n}(i)\right\|^{2}$, where $\left(\boldsymbol{a}_{n}(i)\right)_{n \in \mathbb{N}}$ is the sequence generated by the $i$-th realization of Alg. 1, and $\tau:=100$ is the number of independent realizations in order to smooth out the obtained performance curves. 


\subsection{Employing time-invariant thresholding operators}

By the modifier "time-invariant", we mean that the user-defined parameter $\lambda$ in (5) remains fixed for all the time instants $n \in \mathbb{N}$. The performance of all the employed methods is given in Fig. 3a. In all cases, $K:=K_{*}$. The regularization parameter $\lambda$ was optimized leading to the values shown in the corresponding figure legend. Moreover, APGT-SCAD, without being considerably sensitive to parameter $\alpha$, appeared to perform best when adopting the relatively large value $\alpha=12$.

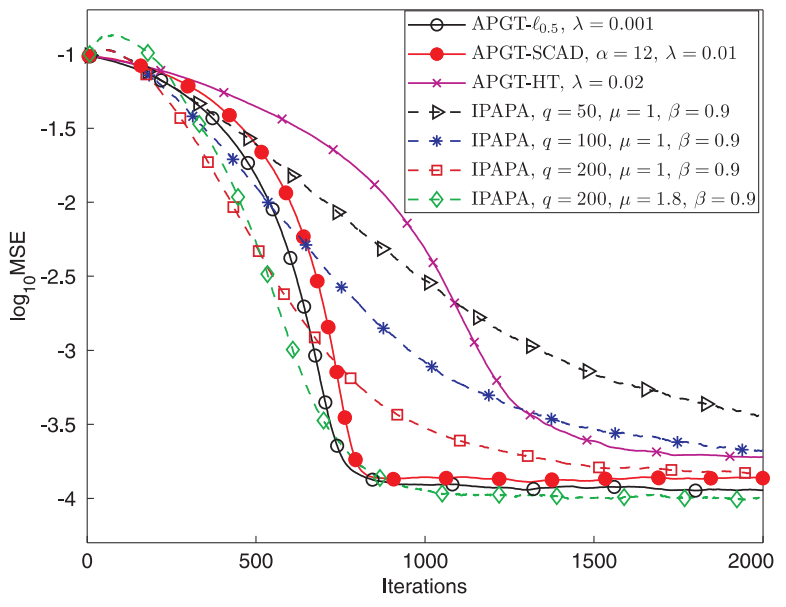

(a) Time-invariant thresholding, i.e., fixed $\lambda$.

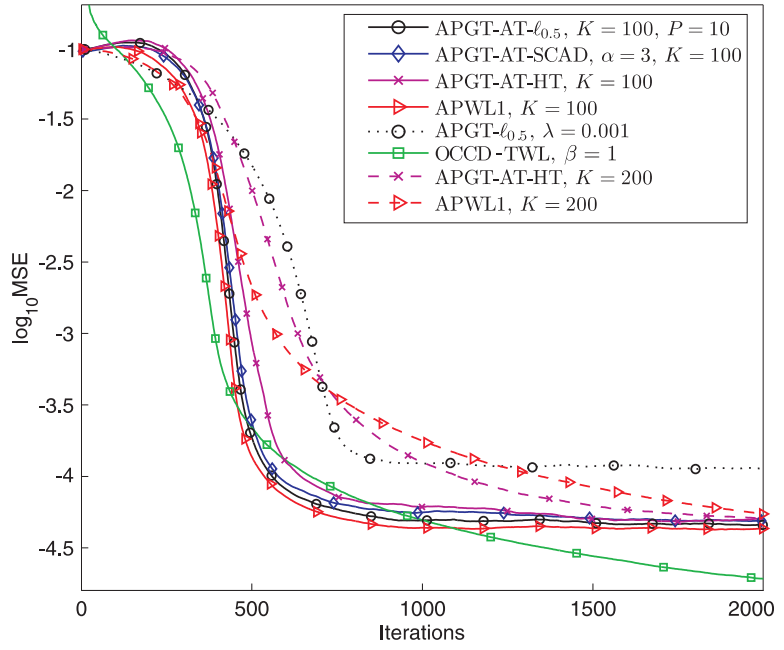

(b) Time-adaptive thresholding, i.e., time-varying $\lambda$.

Figure 3: (a) Performance study of APGT using thresholding operators which are fixed in each iteration and comparison with IPAPA algorithm. (b) Performance study of APGT using thresholding operators which are changing in each iteration and comparison with LASSO solution.

For comparison, the Improved Proportionate Adaptive Projection Algorithm (IPAPA), described in [35, 36], is employed. The projection order of the IPAPA, which plays a similar role to $q$, and therefore the same notation is used, is the major factor which dictates its performance. Dashed curves indicated with triangles, stars and squares correspond to values of $q$ equal to 50, 100, and 200, respectively. The step parameter of the IPAPA is denoted by $\mu$. The best IPAPA performance, i.e., the one depicted with a dashed curve with diamonds, is achieved with $q=200$ and $\mu=1.8$. For lower $q$ values, such a large $\mu$ led to unstable performance. In all cases, the parameter $\beta$, which tunes the weights in the proportionate algorithm 1 , was given the large value $\beta=0.9$ in order to exhibit enhanced sparsity promoting behavior. When larger $q$ values are used, e.g., $q=400$, the performance turned to become somewhat faster, but with a quite elevated steady-state error floor, so the corresponding performance curves are not shown. Moreover, a set-membership counterpart of IPAPA [34] was also examined. This algorithm performed similarly to IPAPA, so the results are not shown to ease visualization. It is clear that the APGT- $\ell_{0.5}$ performs as well as IPAPA. However, this is achieved under a significantly lower computational burden, as will be discussed in Section 5.5 .

\subsection{Employing time-adaptive thresholding operators}

In the previous section, the exact shape of the thresholding function was determined in advance using fixed values for the associated parameters, e.g., $\lambda, \gamma, \alpha$, etc. This is quite limiting, since the proposed

\footnotetext{
${ }^{1}$ See parameter $\alpha$ in (2) of [36]. We call it here $\beta$ in order to avoid confusion with the parameter $\alpha$ of SCAD.
} 
technique has the potential to incorporate time-adaptive a-priori information, in the form of time-varying thresholding operators. This section demonstrates that exploiting this freedom leads APGT to enhanced performance. In particular, $\lambda$ in (5) changes as time $n$ advances. In order to explicitly describe this dependency of $\lambda$ to $n$, we will use hereafter the notation $\lambda_{n}$. Assuming that an estimate $K$ of the true sparsity level $K_{*}$ is available at each iteration $n$, parameter $\lambda_{n}$ is properly tuned in order to guarantee that after thresholding, a fixed number of components will be set equal to zero. With respect to the HT operator, in order to achieve a sparsity level equal to $K$, i.e., $L-K$ components are zero, the quantity $\lambda_{n}$ should be set equal to $\xi_{\boldsymbol{a}_{n}}^{(K)}, \forall n$. For the SCAD case, $\lambda_{n}:=\frac{1}{\alpha} \xi_{\boldsymbol{a}_{n}}^{(K)}, \forall n$, (refer to (9)). In this way, the SCAD shrinkage behavior is preserved and tuned by the user-defined parameter $\alpha$. In a similar manner, an adaptive BT can be built. Going even further, apart from the $K$ larger in magnitude components which remain unaltered, the next, say $P$, smaller in magnitude components could be shrunk according to the bridge rule. This is achieved if we notice that, by definition, $\xi_{\boldsymbol{a}_{n}}^{(K+P)} \leq \xi_{\boldsymbol{a}_{n}}^{(K)}, \forall P \in \overline{1, L-K}$, and that the parameter $\lambda_{n}$ is defined here as the solution of the following equation $\xi_{\boldsymbol{a}_{n}}^{(K+P)}=c_{\mathrm{BT}}\left(\lambda_{n}, \gamma\right)$. In particular, for $\gamma=0.5$, this solution obtains a closed form:

$$
\lambda_{n}=4\left(\frac{\xi_{\boldsymbol{a}_{n}}^{(K+P)}}{3}\right)^{\frac{3}{2}}, \quad \forall n .
$$

For convenience, the full GT operator involving the $\ell_{0.5}$ shrinkage is given next: $\forall i \notin J_{\boldsymbol{x}}^{(K)}$,

$$
z_{i}= \begin{cases}0, & \text { if }\left|x_{i}\right| \leq \xi_{\boldsymbol{a}_{n}}^{(K+P)}, \\ \operatorname{sgn}\left(x_{i}\right)\left(\bar{z}_{i}-\delta\right)_{+}, & \text {if } \xi_{\boldsymbol{a}_{n}}^{(K+P)}<\left|x_{i}\right| \leq \xi_{\boldsymbol{a}_{n}}^{(K)},\end{cases}
$$

where $\bar{z}_{i}$ satisfies $\bar{z}_{i}+\frac{1}{2 \sqrt{\bar{z}_{i}}} \lambda_{n} \operatorname{sgn}\left(z_{i}\right)=\left|x_{i}\right|$, and $\lambda_{n}$ is given by (11).

The performance of APGT methods, using the previous time-adaptive thresholding strategy, hereafter abbreviated as APGT-AT, is shown in Fig. 3b, For reference, the dotted curve marked with open circles is the one from Fig. 3a corresponding to the best APGT method with a fixed $\lambda$. Moreover, the best results for the APGT-AT- $\ell_{0.5}$ are obtained when $P$ assumes a small integer value, such as 10. A conclusion that can be easily drawn is that the incorporation of adaptive thresholding led to a performance boost. Moreover, the performance achieved depends on the thresholding operator that is adopted, with the BT leading to somewhat faster convergence speed compared to SCAD and HT. The performance of APWL1, proposed in [11, is also shown with solid line marked with triangles. It appears that the newly proposed algorithms, and especially APGT-AT- $\ell_{0.5}$, succeeds in achieving a similar convergence behavior and speed compared to APWL1 and, as it will be discussed in Section 5.5. with half the computational complexity. For completeness, the Online Cyclic Coordinate Descent - Time Weighted Lasso (OCCD-TWL), presented in [7, is depicted with solid line marked with squares. The latter is an online algorithm approximating the LASSO problem solution. It is observed, that APGT $(q=390)$, demonstrates a performance competitive to OCCD-TWL, which is an $\mathcal{O}\left(L^{2}\right)$ complexity algorithm.

The advantages of the APGT algorithm over the APWL1 are not limited to the performance improvements and/or to computational complexity savings. The proposed theoretical framework is general enough in order to include other thresholding operators as well, either existing or newly defined. However, the scope of this paper is not a simulation study of all these alternatives of thresholding, and such a route will be studied elsewhere. For example, in [47], implementations of the proposed scheme driven by a different set of PLSTOs, suitable for low complexity operation, and a novel specially customized thresholding operator are presented. In that case, comparison with linear complexity sparsity inducing 
algorithms, such as the Reweighted Zero Attracting-Least Mean Square (RZA-LMS) [6], $\ell_{0}$-LMS [12], and the Sparse Adaptive Orthogonal Matching Pursuit (SpAdOMP) 9] is made in more advanced scenarios, such as system identification with correlated input signal (see [47]) and sparse signal estimation corrupted by non-symmetric and/or impulsive noise.

\subsection{Robustness against inaccurate sparsity level estimates}

With the aid of Fig. 4a, the effect of over- and under-estimation of $K_{*}$ is discussed for the reduced complexity case of $q=20$. We choose a low value for $q$, since we noticed that such a scenario reveals more distinctly the performance sensitivity and related behavior of the APGT with over- or underestimations of $K_{*}$. Moreover, the use of a low value of $q$, reveals the performance advantages of the GT, compared to other linear complexity algorithms, such as the $\ell_{0}$-LMS [12]. As it is seen from the Fig. 4a, the use of the GT mapping results in enhanced performance w.r.t. both APWL1 and $\ell_{0}$-LMS, where the latter was fine-tuned for best convergence speed/error floor trade off. In order to have a reference of the performance achieved when the true sparsity level is given, the APGT-AT- $\ell_{0.5}$ with $K=100$, is also provided in Fig. 4a, Let us start with the under-estimation case and assume that $K=80$, i.e., $20 \%$ lower compared to the true sparsity level. Let us take, for example, the APGT-AT-SCAD curve, which shows an elevated error floor. Notice that the case of under-estimations of $K_{*}$ is not supported theoretically by Thm. 1. With respect to over-estimation, APGT is shown to be very robust. For example, let us see the case where $K_{*}$ is over-estimated by $100 \%$, i.e., $K:=2 K_{*}$. The performance achieved by APGT-AT- $\ell_{0.5}$ (solid line with open circles) is still much better compared to the APWL1, even if APWL1 uses an accurate estimate for the $K_{*}$. Moreover, the degradation resulted from such a large over-estimation appears to be limited. Remarkably, in this low $q$ case, both APGT-AT-HT and APGT-AT-SCAD, drawn with solid lines marked with x-crosses and diamonds, respectively, have benefited from the over-estimation. The reason for this is that when $q$ is small, the tentative estimates of the unknown vector in each iteration are likely to be not accurate enough in order for the $K_{*}$ larger of them to reveal the true support of the vector. An over-estimated $K_{*}$ leads to less strict HT and SCAD thresholding operators, which allow components that would otherwise be set equal to zero, to survive.

All the results above have been confirmed with higher levels of over-estimation.

The results are similar when the algorithms operate with higher complexity, i.e., $q=390$, with the difference that the performance of APGT-AT-HT and APGT-AT-SCAD does not benefited as much as previously by an over-estimation of $K$. The APGT-AT-SCAD and APGT-AT- $\ell_{0.5}$ perform similarly, so the corresponding curves are not shown. A thorough examination of several scenarios, in the case where $q$ attains low values, is deferred to a future work.

\subsection{Tracking ability of the APGT}

Fig. $4 \mathrm{~b}$ shows the ability of the tested algorithms to track an abrupt change of the unknown vector $\boldsymbol{a}_{*}$, which is realized here after 1500 observations is examined. This is a typical setting used in adaptive filtering [41] community to study the tracking agility of an algorithm. Here, in order to give an essence from the CS paradigm, we consider the vector to be not sparse itself but to have a sparse wavelet representation. In the first half, the signal under consideration is of length $L=1024$, with $K_{*}=100$ non zero wavelet coefficients. However, at the 1500 time instant, ten randomly selected wavelet coefficients change their values from 0 to a randomly selected nonzero one. Since the sparsity level of the signal changes (from 100 to 110, at most) and it is not possible to know $K_{*}$ exactly in advance, taking into account that the methods we propose are quite robust to $K_{*}$ over-estimations, we set $K=150$ throughout the whole experiment. Moreover, $q$ is set to 390 . 


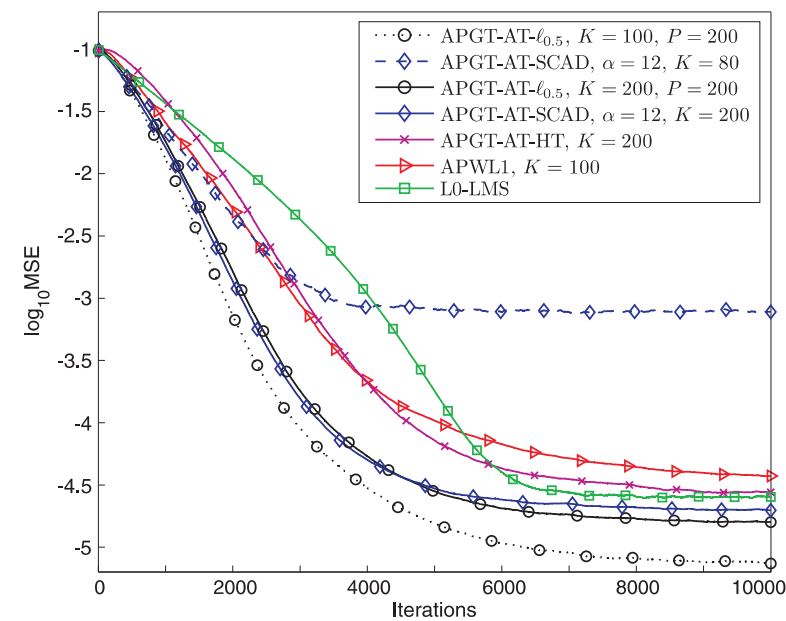

(a) Robustness against erroneous estimates of the sparsity level.

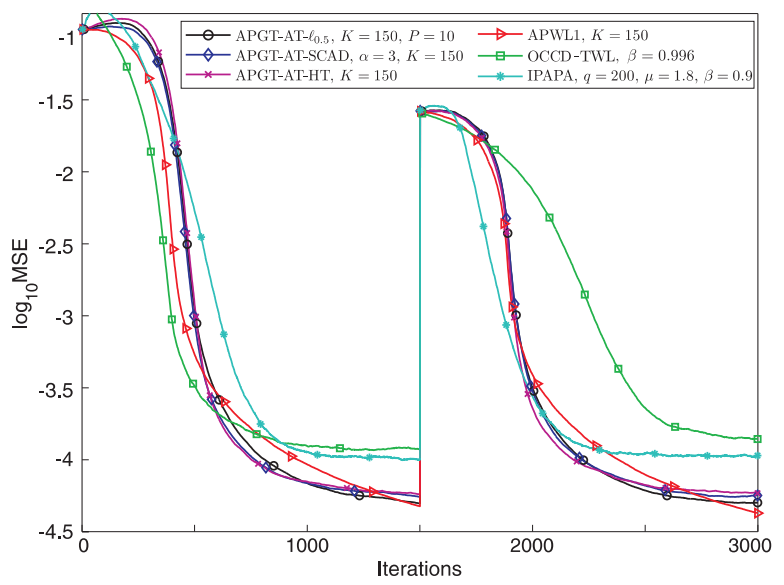

(b) Robustness against time variations of the desired solution.

Figure 4: (a) Robustness of APGT-AT in the cases of an under-estimation and an over-estimation of $K_{*}=100$, i.e., $K=80$ and $K=200$, respectively. The $q=20$ in these experiments. (b) The unknown vector has a sparse wavelet representation which changes abruptly after the reception of 1500 observations.

For the OCCD-TWL, an RLS-like forgetting factor lower than 1 is adopted, in order to succeed in re-estimating the unknown signal after the abrupt change. More specifically, the value of 0.996 appeared to offer a good trade-off between convergence speed and steady-state error floor. However, the OCCDTWL convergence speed slows down after the 1500 time instant, something which was observed and discussed in [11] as well. The IPAPA method, catches up quickly after the abrupt change; however, the attained error floor is higher than that of the APGT.

\subsection{Computational complexity}

The choice of the thresholding operator affects significantly the overall computational burden for two reasons. First, the thresholding function itself requires a larger or smaller number of mathematical operations depending on the specific thresholding rule. Such operations can be multiplications, divisions, as well as sorting operations. Additions are ignored since they are considered to be much less costly. A second attribute of the thresholding rule, which affects complexity, is whether its outcome is a sparse vector with a certain sparsity level or not. Indeed, if the thresholding operator produces vectors which are, say, $\bar{K}$-sparse, then projections in APGT involve inner products with sparse vectors where the number of required multiplications equal to $\bar{K}$ instead of $L$. The HT and the GT with Bridge$\ell_{0.5}$ shrinkage function, as they where presented in 5.2, belong to this category with $\bar{K}=K$ and $\bar{K}=K+P$, respectively. The SCAD thresholding rule does not guarantee a fixed number of zeros after its application. This is also the case of the APWL1 [11. Moreover, in the case of the APWL1, exact projections onto the weighted $\ell_{1}$-ball need to be computed, and in order to do so, the sorting of a vector is necessary, which requires in general $\mathcal{O}\left(L \log _{2} L\right)$ operations. However, by adopting a divideand-conquer approach, as in [49], one might reduce the above computational complexity down to $O(L)$ operations.

The worst-case computational complexities of all the methods employed are given in Table 1. The parameter $e_{1}$ is either 1 or 2 , depending on whether all $\omega_{i}^{(n)}$ of the APGT are given the same value or 


\begin{tabular}{|c|c|c|c|c|}
\hline \multirow[t]{2}{*}{ Methods } & \multicolumn{4}{|l|}{ Operations } \\
\hline & Multiplications & Divisions & Sortings & Powers \\
\hline APGT-AT-HT & $\left(q e_{1}+e_{2}+1\right) L+\left(K+e_{1}+1\right) q$ & $e_{2}+1$ & $\mathcal{O}(L)$ & - \\
\hline APGT-AT- $\ell_{0.5}$ & $\left(q e_{1}+e_{2}+1\right) L+\left(K+P+e_{1}+1\right) q+12 P+1$ & $P+e_{2}+2$ & $\mathcal{O}(L)$ & $3 P+1$ \\
\hline APGT-AT-SCAD & $\left(q e_{1}+e_{2}+1\right) L+\left(L+e_{1}+1\right) q+(L-K)$ & $L-K+e_{2}+1$ & $\mathcal{O}(L)$ & - \\
\hline APWL1 & $\left(q e_{1}+e_{2}+1\right) L+\left(L+e_{1}+1\right) q+3 L$ & $2 L+e_{2}+1$ & $\mathcal{O}(L)$ & - \\
\hline OCCD-TWL & $3 L^{2}+3 L$ & $L$ & - & - \\
\hline IPAPA & $\mathcal{O}\left(q^{3}\right)+\left(q^{2}+3 q+1\right) L+q$ & - & - & - \\
\hline
\end{tabular}

Table 1: Computational complexities of all the methods employed.

not. In the examples of this paper the former is the case, i.e., $e_{1}=1$. Moreover, parameter $e_{2}$ is either 1 , if the $\ell_{2}$ norm of the input vectors $\left(\boldsymbol{u}_{n}\right)_{n \in \mathbb{N}}$ is arbitrary, or 0 , if it is normalized to unity.

\section{Conclusions}

The present paper contributed to sparsity-aware online learning tasks in the following three ways: (i) it established a Generalized Thresholding (GT) mapping, which can incorporate as a shrinkage function the majority of the thresholding rules found in the literature, (iii) it proposed a non-convexly constrained, online learning algorithm for sparse signal recovery tasks with a computational complexity which scales linearly to the number of unknowns, and (iii) it introduced a family of mappings which serves as the wide functional analytic stage for the study of the previous GT operator. Rigorous discussions on the properties of all the previous functional analytic tools, as well as a convergence analysis of the proposed algorithm were provided. To validate the theoretical findings regarding our algorithm, extensive experiments were conducted, which showed that the proposed methodology offers a sound theoretical, and very competitive time-adaptive technique, with lower computational complexity than several of the state-of-the-art, sparsity-promoting, online learning algorithms.

\section{A Convex Sets, Convex Functions, and Projection Mappings}

A subset $C$ of $\mathbb{R}^{L}$ will be called convex, if for any $\boldsymbol{a}, \boldsymbol{a}^{\prime} \in C$, the line segment $\left\{\lambda \boldsymbol{a}+(1-\lambda) \boldsymbol{a}^{\prime}\right.$ : $\lambda \in[0,1]\}$ lies in $C$. A function $\Theta: \mathbb{R}^{L} \rightarrow \mathbb{R}$ is called convex if $\forall \boldsymbol{a}, \boldsymbol{a}^{\prime} \in \mathbb{R}^{L}$, and $\forall \lambda \in[0,1]$, we have $\Theta\left(\lambda \boldsymbol{a}+(1-\lambda) \boldsymbol{a}^{\prime}\right) \leq \lambda \Theta(\boldsymbol{a})+(1-\lambda) \Theta\left(\boldsymbol{a}^{\prime}\right)$. The 0 -th level set of the convex $\Theta$ is defined as $\operatorname{lev}_{\leq 0}(\Theta):=\left\{\boldsymbol{a} \in \mathbb{R}^{L}: \Theta(\boldsymbol{a}) \leq 0\right\}$. A subgradient of the convex function $\Theta$ at a point $\boldsymbol{a}$, denoted as $\Theta^{\prime}(\boldsymbol{a})$, is an $L$-dimensional vector such that $(\boldsymbol{v}-\boldsymbol{a})^{\top} \Theta^{\prime}(\boldsymbol{a})+\Theta(\boldsymbol{a}) \leq \Theta(\boldsymbol{v}), \forall \boldsymbol{v} \in \mathbb{R}^{L}$. In general, the number of the subgradients of $\Theta$ at $\boldsymbol{a}$ is infinite. The set of all subgradients of $\Theta$ at a point $\boldsymbol{a}$ is called subdifferential, and it is denoted by $\partial \Theta(\boldsymbol{a})$. In the case where $\Theta$ is differentiable at $\boldsymbol{a}$, then the subgradient $\Theta^{\prime}(\boldsymbol{a})$ is unique, and it is nothing but the gradient of $\Theta$ at $\boldsymbol{a}$.

Given a closed convex $C \subset \mathbb{R}^{L}$, define the (metric) distance function $d(\cdot, C): \mathbb{R}^{L} \rightarrow \mathbb{R}$ to $C$ as follows: $\forall \boldsymbol{a} \in \mathbb{R}^{L}, d(\boldsymbol{a}, C):=\inf \{\|\boldsymbol{a}-\boldsymbol{v}\|: \boldsymbol{v} \in C\}$. Notice that $d(\cdot, C)$ is convex with $\operatorname{lev}_{\leq 0} d(\cdot, C)=C$. The (metric) projection onto $C$ is defined as the mapping $P_{C}: \mathbb{R}^{L} \rightarrow C$, which maps an $\boldsymbol{a} \in \mathbb{R}^{L}$ to the unique $P_{C}(\boldsymbol{a}) \in C$, such that $\left\|\boldsymbol{a}-P_{C}(\boldsymbol{a})\right\|=d(\boldsymbol{a}, C)$. For example, the subdifferential of $d(\cdot, C)$ is given as follows:

$$
\partial d(\boldsymbol{a}, C)= \begin{cases}N_{C}(\boldsymbol{a}) \cap B[0,1], & \text { if } \boldsymbol{a} \in C, \\ \frac{\boldsymbol{a}-P_{C}(\boldsymbol{a})}{d(\boldsymbol{a}, C)}, & \text { if } \boldsymbol{a} \notin C,\end{cases}
$$


where $N_{C}(\boldsymbol{a}):=\left\{\boldsymbol{v} \in \mathbb{R}^{L}: \boldsymbol{v}^{\top}(\boldsymbol{y}-\boldsymbol{a}) \leq 0, \forall \boldsymbol{y} \in C\right\}$.

\section{B The Penalized Least-Squares Task}

Going back to (11), choose $N \in \mathbb{N}_{*}$, and define $\boldsymbol{U}_{n}:=\left[\boldsymbol{u}_{n}, \boldsymbol{u}_{n-1}, \ldots, \boldsymbol{u}_{n-N+1}\right] \in \mathbb{R}^{L \times N}$, as well as $\boldsymbol{y}_{n}:=\left[y_{n}, y_{n-1}, \ldots, y_{n-N+1}\right]^{\top} \in \mathbb{R}^{N}$, and $\boldsymbol{v}_{n}:=\left[v_{n}, v_{n-1}, \ldots, v_{n-N+1}\right]^{\top} \in \mathbb{R}^{N}$. Then, it can be easily verified that (10) takes the form of $\boldsymbol{y}_{n}=\boldsymbol{U}_{n}^{\top} \boldsymbol{a}_{*}+\boldsymbol{v}_{n}, \forall n \in \mathbb{N}$. The mainstream of the batch sparsitypromoting algorithms utilize all the gathered $N$ training data to find an exact or approximate solution, in most cases iteratively, to the following penalized least-squares minimization task,

$$
\min _{\boldsymbol{a} \in \mathbb{R}^{L}} \frac{1}{2}\left\|\boldsymbol{y}_{n}-\boldsymbol{U}_{n}^{\top} \boldsymbol{a}\right\|^{2}+\lambda \sum_{i=1}^{L} p\left(\left|a_{i}\right|\right),
$$

where $p: \mathbb{R} \rightarrow[0, \infty)$ stands for a sparsity-promoting and non-convex, in general, penalty function, $\lambda \in(0, \infty)$ is the regularization parameter, and $a_{i}$ stands for the $i$-th coordinate of the vector $\boldsymbol{a}$.

Choices for $p$ are numerous; if, for example, $p(|a|):=\chi_{\mathbb{R} \backslash\{0\}}(|a|), \forall a \in \mathbb{R}$, where $\chi_{\mathscr{A}}$ stands for the characteristic function with respect to $\mathscr{A} \subset \mathbb{R}$, i.e., $\chi_{\mathscr{A}}(\alpha):=1$, if $\alpha \in \mathscr{A}$, and $\chi_{\mathscr{A}}(\alpha):=0$, if $\alpha \notin \mathscr{A}$, then the regularization term $\sum_{i=1}^{L} p\left(\left|a_{i}\right|\right)$ becomes the $\ell_{0}$-norm of $\boldsymbol{a}$. In the case where $p(|a|):=|a|$, $\forall a \in \mathbb{R}$, then the regularization term is nothing but the $\ell_{1}$-norm $\|a\|_{1}:=\sum_{i=1}^{L}\left|a_{i}\right|$, and the task (14) becomes the celebrated LASSO [14]. However, it has been observed that if some of the LASSO's regularity conditions are violated, then LASSO is sub-optimal for model selection [13, 20, 22, 24, 27. Such a behavior has motivated the search for non-convex penalty functions $p$, which bridge the gap between the $\ell_{0^{-}}$and $\ell_{1}$-norm; for example, the $\ell_{\gamma}$ penalty, for $\gamma \in(0,1)$, 13], the log [18, the SCAD [18,19], the MC+ [24,27], and the transformed $\ell_{1}$ [18] penalties.

Recently, sparsity-promoting coordinate-wise optimization techniques for solving the task (14) are attracting a lot of interest [7, 26, 27]. To be more concrete, assume, for example, that $N=L$, and that the matrix $\boldsymbol{U}_{n}$ is orthogonal. Byy defining $\tilde{\boldsymbol{a}}_{n}:=\boldsymbol{U}_{n} \boldsymbol{y}_{n}$, (14) can be equivalently viewed as the following separable optimization task [18,21],

$$
\min _{\boldsymbol{a} \in \mathbb{R}^{L}} \sum_{i=1}^{L} \frac{1}{2 \lambda}\left(\tilde{a}_{i}-a_{i}\right)^{2}+p\left(\left|a_{i}\right|\right) .
$$

Under some mild regularity conditions on $p$ [18], the minimization task of (15) possesses a unique minimizer. Due to the separability of (15) in coordinates, the minimization task of (15) can be viewed as a task defined on an 1-dimensional axis, instead of an $L$-dimensional domain. Accordingly, the problem reduces to the univariate PLS task described in (4).

Figs. 2(b-d), show the thresholding functions (PLSTO, see (5)), which solve (4) for some of the most commonly employed penalty functions. For example, if $p(|a|):=\left[\lambda^{2}-(|a|-\lambda)^{2} \chi_{[0, \lambda)}(|a|)\right] / \lambda$, $\forall a \in \mathbb{R}$, then the resulting PLSTO is the celebrated Hard Thresholding (HT) mapping [18], which is depicted in Fig. 2a together with the well-known Soft Thresholding (ST) mapping which results in the case where $p(|a|):=|a|$, i.e. is chosen such that to lead to the LASSO task. Note that both ST and HT operators have been effectively employed in iterative thresholding schemes for fast sparse signal recovery under the compressed sensing framework [3, 5, 50]. The rest of the thresholding rules, shown in Fig. 2b correspond to the MC+ penalty [24,27] and the SCAD [19], respectively. Both SCAD and $\mathrm{MC}+$ leave large components unchanged, like HT, while avoiding being discontinuous and at the same time allowing a linear/gradual transition between the "kill" and the "keep" areas of HT. HT is far from 
being the only discontinuous thresholding operator. An example is shown in Figs. 2k, by the widely known Bridge threshold [13], which is related to the $\ell_{\gamma}$ penalty, $\gamma<1$ [51]. Note that this thresholding rule comprise nonlinear segments. Continuous thresholding functions, that contain nonlinear parts, are shown in Fig. 2(d). More specifically, the non-negative garrote [16] and representatives of the n-degree garrote threshold are shown. Similar thresholding functions are also the hyperbolic shrinkage rule [17] and PLSTO's stemming from the nonlinear diffusive filtering approach [21].

\section{Properties of the GT Mapping}

\section{Theorem 2.}

1. $\forall \boldsymbol{x} \in \mathbb{R}^{L}, J_{T_{\mathrm{GT}}^{(K)}(\boldsymbol{x})}^{(K)}=J_{\boldsymbol{x}}^{(K)}$.

2. $\operatorname{Fix}\left(T_{\mathrm{GT}}^{(K)}\right)=\bigcup_{J \in \mathscr{T}(K, L)} M_{J}$. Notice, here, that $\operatorname{Fix}\left(T_{\mathrm{GT}}^{(K)}\right)$, as a union of subspaces, is non-convex.

3. Let a sequence $\left(\boldsymbol{x}_{n}\right)_{n \in \mathbb{N}} \subset \mathbb{R}^{L}$ and an $\boldsymbol{x}_{*} \in \mathbb{R}^{L}$. If $\lim _{n \rightarrow \infty} \boldsymbol{x}_{n}=\boldsymbol{x}_{*}$, and $\lim _{n \rightarrow \infty}\left(I-T_{\mathrm{GT}}^{(K)}\right)\left(\boldsymbol{x}_{n}\right)=\mathbf{0}$, then $\boldsymbol{x}_{*} \in \operatorname{Fix}\left(T_{\mathrm{GT}}^{(K)}\right)$. This property can be rephrased as $I-T_{\mathrm{GT}}^{(K)}$ being demiclosed at $\mathbf{0}$ [28].

4. $T_{\mathrm{GT}}^{(K)}$ is 1-attracting partially quasi-nonexpansive, i.e., $\forall \boldsymbol{x} \in \mathbb{R}^{L}, \forall \boldsymbol{y} \in M_{J_{\boldsymbol{x}}^{(K)}},\left\|\boldsymbol{x}-T_{\mathrm{GT}}^{(K)}(\boldsymbol{x})\right\|^{2} \leq$ $\|\boldsymbol{x}-\boldsymbol{y}\|^{2}-\left\|T_{\mathrm{GT}}^{(K)}(\boldsymbol{x})-\boldsymbol{y}\right\|^{2}$.

Proof:

1. Define $\boldsymbol{z}:=T_{\mathrm{GT}}^{(K)}(\boldsymbol{x})$. In order to derive a contradiction, assume that $J_{\boldsymbol{x}}^{(K)} \neq J_{\boldsymbol{z}}^{(K)}$. Since both $J_{\boldsymbol{x}}^{(K)}, J_{\boldsymbol{z}}^{(K)}$ have the same cardinality, the previous assumption means that there exist $l_{0}, l_{0}^{\prime}$ such that $l_{0} \in J_{\boldsymbol{x}}^{(K)} \backslash J_{\boldsymbol{z}}^{(K)}$, and $l_{0}^{\prime} \in J_{\boldsymbol{z}}^{(K)} \backslash J_{\boldsymbol{x}}^{(K)}$. Hence, $\left|x_{l_{0}^{\prime}}\right|=\left|\operatorname{shr}\left(x_{l_{0}^{\prime}}\right)\right|=\left|z_{l_{0}^{\prime}}\right| \geq\left|z_{l_{0}}\right|=\left|x_{l_{0}}\right| \geq\left|x_{l_{0}^{\prime}}\right|$. The previous result implies that $\left|x_{l_{0}}\right|=\left|x_{l_{0}^{\prime}}\right|$, which, in turn, suggests by the definition of $J_{\boldsymbol{x}}^{(K)}$ that $l_{0}<l_{0}^{\prime}$. Moreover, $\left|z_{l_{0}^{\prime}}\right|=\left|z_{l_{0}}\right|$ and $l_{0}^{\prime}<l_{0}$ by the definition of $J_{\boldsymbol{z}}^{(K)}$. Thus, $l_{0}<l_{0}^{\prime}<l_{0}$, which is absurd. This contradiction establishes the claim of Thm. 211

2. Pick any $\boldsymbol{x} \in \bigcup_{J \in \mathscr{T}(K, L)} M_{J}$. It is easy to verify by Def. 3 that $T_{\mathrm{GT}}^{(K)}(\boldsymbol{x})=\boldsymbol{x}$, i.e., $\boldsymbol{x} \in \operatorname{Fix}\left(T_{\mathrm{GT}}^{(K)}\right)$. To prove the opposite inclusion, assume any $\boldsymbol{x} \in \operatorname{Fix}\left(T_{\mathrm{GT}}^{(K)}\right)$, i.e., $T_{\mathrm{GT}}^{(K)}(\boldsymbol{x})=\boldsymbol{x}$. Since $\forall l \in J_{\boldsymbol{x}}^{(K)}$, the relation $T_{\mathrm{GT}}^{(K)}(\boldsymbol{x})=\boldsymbol{x}$ leads to the trivial result $x_{l}=x_{l}$, we deal here only with the more interesting case of $l \notin J_{\boldsymbol{x}}^{(K)}$. For such an $l$, according to Def. 3, we must have $\operatorname{shr}\left(x_{l}\right)=x_{l}$, which implies that $\left|\operatorname{shr}\left(x_{l}\right)\right|=\left|x_{l}\right|$. However, by the properties of shr, given in Defs. 35.5and 36, we necessarily obtain that $x_{l}=0$. Since this holds $\forall l \notin J_{\boldsymbol{x}}^{(K)}$, Def. 2 suggests that $\boldsymbol{x} \in M_{J_{\boldsymbol{x}}^{(K)}}$. Now, recall that $J_{\boldsymbol{x}}^{(K)} \in \mathscr{T}(K, L)$ to establish the inclusion $\boldsymbol{x} \in \bigcup_{J \in \mathscr{T}(K, L)} M_{J}$.

3. (a) Assume, for a contradiction, that there exists an $\varepsilon>0$ and a subsequence $\left(n_{k}\right)_{k \in \mathbb{N}}$, such that $\left|x_{n_{k}, l_{n_{k}}}\right| \geq \varepsilon, \forall l_{n_{k}} \notin J_{\boldsymbol{x}_{n_{k}}}^{(K)}, \forall k \in \mathbb{N}$.

By Def. 36, $\exists \delta>0$ such that $\left|\operatorname{shr}\left(x_{n_{k}, l_{n_{k}}}\right)\right| \leq\left|x_{n_{k}, l_{n_{k}}}\right|-\delta, \forall k$. Then, it is easy to verify that $\forall k$, $\left|x_{n_{k}, l_{n_{k}}}-\operatorname{shr}\left(x_{n_{k}, l_{n_{k}}}\right)\right| \geq\left|x_{n_{k}, l_{n_{k}}}\right|-\left|\operatorname{shr}\left(x_{n_{k}, l_{n_{k}}}\right)\right| \geq\left|x_{n_{k}, l_{n_{k}}}\right|-\left|x_{n_{k}, l_{n_{k}}}\right|+\delta=\delta$. This implies that $\forall k$,

$$
\sum_{l \notin J_{\boldsymbol{x}_{n_{k}}}^{(K)}}\left(x_{n_{k}, l}-\operatorname{shr}\left(x_{n_{k}, l}\right)\right)^{2} \geq\left(x_{n_{k}, l_{n_{k}}}-\operatorname{shr}\left(x_{n_{k}, l_{n_{k}}}\right)\right)^{2} \geq \delta^{2} .
$$


Notice that $\left\|\left(I-T_{\mathrm{GT}}^{(K)}\right)\left(\boldsymbol{x}_{n}\right)\right\|^{2}=\sum_{l \notin J_{\boldsymbol{x}_{n}}^{(K)}}\left(x_{n, l}-\operatorname{shr}\left(x_{n, l}\right)\right)^{2}$. Hence, the assumption that $\lim _{n \rightarrow \infty}(I-$ $\left.T_{\mathrm{GT}}^{(K)}\right)\left(\boldsymbol{x}_{n}\right)=\mathbf{0}$ implies that for the $\delta$ of (16) $), \exists n_{0} \in \mathbb{N}$ such that $\forall n \geq n_{0}, \sum_{l \notin J_{\boldsymbol{x}_{n}}^{(K)}}\left(x_{n, l}-\operatorname{shr}\left(x_{n, l}\right)\right)^{2}<\delta^{2}$. This contradicts (16). In other words, our initial claim is wrong, and the contrary proposition becomes: $\forall \varepsilon>0$, there exists an $n_{0} \in \mathbb{N}$ such that $\left|x_{n, l}\right|<\varepsilon, \forall l \notin J_{\boldsymbol{x}_{n}}^{(K)}, \forall n \geq n_{0}$. This can be equivalently written in a more compact form as follows:

$$
\lim _{n \rightarrow \infty} \max \left\{\left|x_{n, l}\right|: l \notin J_{\boldsymbol{x}_{n}}^{(K)}\right\}=0 .
$$

(b) Let us define here

$$
J_{\infty}:=\liminf _{n \rightarrow \infty} J_{\boldsymbol{x}_{n}}^{(K)}:=\bigcup_{n=0}^{\infty} \bigcap_{m=n}^{\infty} J_{\boldsymbol{x}_{m}}^{(K)} .
$$

In words, $J_{\infty}$ contains all those points which belong to all but a finite number of $J_{\boldsymbol{x}_{n}}^{(K)}$ s. There are two cases regarding $J_{\boldsymbol{x}_{*}}^{(K)}$ and $J_{\infty}$; either $J_{\boldsymbol{x}_{*}}^{(K)} \cap J_{\infty} \neq \emptyset$ or $J_{\boldsymbol{x}_{*}}^{(K)} \cap J_{\infty}=\emptyset$. Notice that the latter covers also the case where $J_{\infty}=\emptyset$. Let us examine each case separately.

i. The case of $J_{\boldsymbol{x}_{*}}^{(K)} \cap J_{\infty} \neq \emptyset$.

A. Assume that $J_{\boldsymbol{x}_{*}}^{(K)} \subset J_{\infty}$. This implies that there exists an $n_{0}$ such that $J_{\boldsymbol{x}_{*}}^{(K)} \subset \bigcap_{n \geq n_{0}} J_{\boldsymbol{x}_{n}}^{(K)}$. Since both $J_{\boldsymbol{x}_{*}}^{(K)}$ and $J_{\boldsymbol{x}_{n}}^{(K)}$ have the same cardinality, i.e., $K$, we obtain that $\forall n \geq n_{0}, J_{\boldsymbol{x}_{*}}^{(K)}=J_{\boldsymbol{x}_{n}}^{(K)}$. Choose any $l \notin J_{\boldsymbol{x}_{*}}^{(K)}=J_{\boldsymbol{x}_{n}}^{(K)}$. By (17), $\lim _{n \rightarrow \infty} x_{n, l}=0=x_{*, l}$. Thus, $\forall l \notin J_{\boldsymbol{x}_{*}}^{(K)}, x_{*, l}=0$, or equivalently, $\boldsymbol{x}_{*} \in \bigcup_{J \in \mathscr{T}(K, L)} M_{J}$.

B. Assume now that $J_{\boldsymbol{x}_{*}}^{(K)} \not \subset J_{\infty}$. Hence, there exists an $l \in J_{\boldsymbol{x}_{*}}^{(K)}$ and a subsequence $\left(n_{k}\right)_{k \in \mathbb{N}}$ such that $l \notin J_{\boldsymbol{x}_{n}}^{(K)}, \forall k \in \mathbb{N}$. By (17), $\lim _{n \rightarrow \infty} x_{n, l}=0=x_{*, l}$. Since $l \in J_{\boldsymbol{x}_{*}}^{(K)}$, we clearly have that $x_{*, l^{\prime}}=0$, $\forall l^{\prime} \notin J_{\boldsymbol{x}_{*}}^{(K)}$. Hence, $\boldsymbol{x}_{*} \in \bigcup_{J \in \mathscr{T}(K, L)} M_{J}$.

ii. The case of $J_{\boldsymbol{x}_{*}}^{(K)} \cap J_{\infty}=\emptyset$. This means that there exists an $l \in J_{\boldsymbol{x}_{*}}^{(K)}$ and a subsequence $\left(n_{k}\right)_{k \in \mathbb{N}}$ such that $l \notin J_{\boldsymbol{x}_{n_{k}}}^{(K)}, \forall k \in \mathbb{N}$. Thus, similarly to our previous arguments, $\boldsymbol{x}_{*} \in \bigcup_{J \in \mathscr{T}(K, L)} M_{J}$.

4. Define $R_{K}:=2 T_{\mathrm{GT}}^{(K)}-I$. Given any $\boldsymbol{x} \in \mathbb{R}^{L}$, let $\boldsymbol{z}:=T_{\mathrm{GT}}^{(K)}(\boldsymbol{x})$, as in Def. 3, Then, verify that $\forall \boldsymbol{y} \in M_{J_{\boldsymbol{x}}^{(K)}}$,

$$
\begin{aligned}
\left\|R_{K}(\boldsymbol{x})-\boldsymbol{y}\right\|^{2} & =\sum_{l=1}^{L}\left(2 z_{l}-x_{l}-y_{l}\right)^{2} \\
& =\sum_{l \in J_{\boldsymbol{x}}^{(K)}}\left(x_{l}-y_{l}\right)^{2}+\sum_{l \notin J_{\boldsymbol{x}}^{(K)}}\left(2 \operatorname{shr}\left(x_{l}\right)-x_{l}\right)^{2} \\
& \leq \sum_{l \in J_{\boldsymbol{x}}^{(K)}}\left(x_{l}-y_{l}\right)^{2}+\sum_{l \notin J_{\boldsymbol{x}}^{(K)}} x_{l}^{2}=\|\boldsymbol{x}-\boldsymbol{y}\|^{2} .
\end{aligned}
$$

The previous inequality is obtained from the observation that the properties of shr in Def. 3 suggest $\operatorname{shr}^{2}\left(x_{l}\right) \leq x_{l} \operatorname{shr}\left(x_{l}\right)$, and from the following elementary calculations: $\left(2 \operatorname{shr}\left(x_{l}\right)-x_{l}\right)^{2}=4 \operatorname{shr}^{2}\left(x_{l}\right)+x_{l}^{2}-$ $4 x_{l} \operatorname{shr}\left(x_{l}\right) \leq 4 \operatorname{shr}^{2}\left(x_{l}\right)+x_{l}^{2}-4 \operatorname{shr}^{2}\left(x_{l}\right)$. Hence, $\forall \boldsymbol{x} \in \mathbb{R}^{L}, \forall \boldsymbol{y} \in M_{J_{\boldsymbol{x}}^{(K)}},\left\|R_{K}(\boldsymbol{x})-\boldsymbol{y}\right\|^{2} \leq\|\boldsymbol{x}-\boldsymbol{y}\|^{2} \Leftrightarrow$ $\left\|2 T_{\mathrm{GT}}^{(K)}(\boldsymbol{x})-\boldsymbol{x}-\boldsymbol{y}\right\|^{2} \leq\|\boldsymbol{x}-\boldsymbol{y}\|^{2} \Leftrightarrow\left\|2\left(T_{\mathrm{GT}}^{(K)}(\boldsymbol{x})-\boldsymbol{y}\right)-(\boldsymbol{x}-\boldsymbol{y})\right\|^{2} \leq\|\boldsymbol{x}-\boldsymbol{y}\|^{2} \Leftrightarrow\left\|\boldsymbol{x}-T_{\mathrm{GT}}^{(K)}(\boldsymbol{x})\right\|^{2} \leq$ 
$\|\boldsymbol{x}-\boldsymbol{y}\|^{2}-\left\|T_{\mathrm{GT}}^{(K)}(\boldsymbol{x})-\boldsymbol{y}\right\|^{2}$, where in order to obtain the last equivalence we used some elementary algebra, and the fact

$2\left\langle\boldsymbol{x}-\boldsymbol{y}, T_{\mathrm{GT}}^{(K)}(\boldsymbol{x})-\boldsymbol{y}\right\rangle=\|\boldsymbol{x}-\boldsymbol{y}\|^{2}+\left\|T_{\mathrm{GT}}^{(K)}(\boldsymbol{x})-\boldsymbol{y}\right\|^{2}-\left\|\boldsymbol{x}-T_{\mathrm{GT}}^{(K)}(\boldsymbol{x})\right\|^{2}$. This establishes the claim of Thm. 24.

\section{Proof of Theorem 1}

Let us define first a sequence of convex functions $\left(\Theta_{n}\right)_{n \in \mathbb{N}}$ in an inductive way. Given the time index $n$, and the estimate $\boldsymbol{a}_{n} \in \mathbb{R}^{L}$, define the following convex function; $\forall \boldsymbol{a} \in \mathbb{R}^{L}$,

$$
\Theta_{n}(\boldsymbol{a}):=\left\{\begin{array}{rr}
\sum_{i \in \mathcal{I}_{n}} \frac{\omega_{i}^{(n)} d\left(\boldsymbol{a}_{n}, S_{i}\left[\epsilon_{i}\right]\right)}{\sum_{j \in \mathcal{I}_{n}} \omega_{j}^{(n)} d\left(\boldsymbol{a}_{n}, S_{j}\left[\epsilon_{j}\right]\right)} d\left(\boldsymbol{a}, S_{i}\left[\epsilon_{i}\right]\right) \\
=\frac{1}{L_{n}} \sum_{i \in \mathcal{I}_{n}} \omega_{i}^{(n)} d\left(\boldsymbol{a}_{n}, S_{i}\left[\epsilon_{i}\right]\right) d\left(\boldsymbol{a}, S_{i}\left[\epsilon_{i}\right]\right), \\
0, & \text { if } \mathcal{I}_{n} \neq \emptyset,
\end{array}\right.
$$

where $L_{n}:=\sum_{j \in \mathcal{I}_{n}} \omega_{j}^{(n)} d\left(\boldsymbol{a}_{n}, S_{j}\left[\epsilon_{j}\right]\right)$. It is easy to verify by the definition of $\mathcal{I}_{n}$, that if $\mathcal{I}_{n} \neq \emptyset$, then $\forall i \in \mathcal{I}_{n}, d\left(\boldsymbol{a}_{n}, S_{i}\left[\epsilon_{i}\right]\right)>0$, and thus $L_{n}>0$. Moreover, if $\mathcal{I}_{n}=\emptyset$, then $\Theta_{n}^{\prime}(\boldsymbol{a})=0, \forall \boldsymbol{a}$.

Let us look closer to $\Theta_{n}$, and especially only the interesting case of $\mathcal{I}_{n} \neq \emptyset$. By standard subgradient calculus, it can be verified by (13) that

$$
\Theta_{n}^{\prime}\left(\boldsymbol{a}_{n}\right)=\frac{1}{L_{n}} \sum_{i \in \mathcal{I}_{n}} \omega_{i}^{(n)} d\left(\boldsymbol{a}_{n}, S_{i}\left[\epsilon_{i}\right]\right) \frac{\boldsymbol{a}_{n}-P_{S_{i}\left[\epsilon_{i}\right]}\left(\boldsymbol{a}_{n}\right)}{d\left(\boldsymbol{a}_{n}, S_{i}\left[\epsilon_{i}\right]\right)}=\frac{1}{L_{n}} \sum_{i \in \mathcal{I}_{n}} \omega_{i}^{(n)}\left(\boldsymbol{a}_{n}-P_{S_{i}\left[\epsilon_{i}\right]}\left(\boldsymbol{a}_{n}\right)\right) .
$$

Thus, whenever $\mathcal{I}_{n} \neq \emptyset$, we have $\Theta_{n}^{\prime}\left(\boldsymbol{a}_{n}\right)=\mathbf{0}$ iff $\sum_{i \in \mathcal{I}_{n}} \omega_{i}^{(n)}\left(\boldsymbol{a}_{n}-P_{S_{i}\left[\epsilon_{i}\right]}\left(\boldsymbol{a}_{n}\right)\right)=\mathbf{0}$. Hence, for some user-defined parameter $\lambda_{n}>0$, it is straightforward to see that

$$
\boldsymbol{a}_{n}-\lambda_{n} \frac{\Theta_{n}\left(\boldsymbol{a}_{n}\right)}{\left\|\Theta_{n}^{\prime}\left(\boldsymbol{a}_{n}\right)\right\|^{2}} \Theta_{n}^{\prime}\left(\boldsymbol{a}_{n}\right)=\boldsymbol{a}_{n}-\lambda_{n} \frac{\sum_{i \in \mathcal{I}_{n}} \omega_{i}^{(n)} d^{2}\left(\boldsymbol{a}_{n}, S_{i}\left[\epsilon_{i}\right]\right)}{\left\|\sum_{i \in \mathcal{I}_{n}} \omega_{i}^{(n)}\left(\boldsymbol{a}_{n}-P_{S_{i}\left[\epsilon_{i}\right]}\left(\boldsymbol{a}_{n}\right)\right)\right\|^{2}} \sum_{i \in \mathcal{I}_{n}} \omega_{i}^{(n)}\left(\boldsymbol{a}_{n}-P_{S_{i}\left[\epsilon_{i}\right]}\left(\boldsymbol{a}_{n}\right)\right) \text {. }
$$

If we let $\lambda_{n}:=\mu_{n} / \mathcal{M}_{n}$, then an examination of ([6), for both the cases of $\mathcal{I}_{n} \neq \emptyset$ and $\mathcal{I}_{n}=\emptyset$, implies that the proposed algorithm can be rephrased as follows; for $\lambda_{n}:=\mu_{n} / \mathcal{M}_{n} \in\left[\varepsilon^{\prime}, 2-\varepsilon^{\prime}\right]$,

$$
\boldsymbol{a}_{n+1}= \begin{cases}T_{\mathrm{GT}}^{(K)}\left(\boldsymbol{a}_{n}-\lambda_{n} \frac{\Theta_{n}\left(\boldsymbol{a}_{n}\right)}{\left\|\Theta_{n}^{\prime}\left(\boldsymbol{a}_{n}\right)\right\|^{2}} \Theta_{n}^{\prime}\left(\boldsymbol{a}_{n}\right)\right), & \Theta_{n}^{\prime}\left(\boldsymbol{a}_{n}\right) \neq \mathbf{0}, \\ T_{\mathrm{GT}}^{(K)}\left(\boldsymbol{a}_{n}\right), & \Theta_{n}^{\prime}\left(\boldsymbol{a}_{n}\right)=\mathbf{0} .\end{cases}
$$

1. Fix any $\boldsymbol{v} \in \Omega_{n}$, and assume that $\Theta_{n}^{\prime}\left(\boldsymbol{a}_{n}\right) \neq 0$. Then, since $\boldsymbol{v} \in M_{J_{\boldsymbol{a}_{n}}^{(K)}} \subset \operatorname{Fix}\left(T_{\mathrm{GT}}^{(K)}\right)$,

$$
\begin{aligned}
\left\|\boldsymbol{a}_{n+1}-\boldsymbol{v}\right\|^{2} & =\left\|T_{\mathrm{GT}}^{(K)}\left(\boldsymbol{a}_{n}-\lambda_{n} \frac{\Theta_{n}\left(\boldsymbol{a}_{n}\right)}{\left\|\Theta_{n}^{\prime}\left(\boldsymbol{a}_{n}\right)\right\|^{2}} \Theta_{n}^{\prime}\left(\boldsymbol{a}_{n}\right)\right)-\boldsymbol{v}\right\|^{2} \\
& \leq\left\|\left(\boldsymbol{a}_{n}-\boldsymbol{v}\right)-\lambda_{n} \frac{\Theta_{n}\left(\boldsymbol{a}_{n}\right)}{\left\|\Theta_{n}^{\prime}\left(\boldsymbol{a}_{n}\right)\right\|^{2}} \Theta_{n}^{\prime}\left(\boldsymbol{a}_{n}\right)\right\|^{2} \\
& \leq\left\|\boldsymbol{a}_{n}-\boldsymbol{v}\right\|^{2}-\lambda_{n}\left(2-\lambda_{n}\right) \frac{\Theta_{n}^{2}\left(\boldsymbol{a}_{n}\right)}{\left\|\Theta_{n}^{\prime}\left(\boldsymbol{a}_{n}\right)\right\|^{2}} \\
& \leq\left\|\boldsymbol{a}_{n}-\boldsymbol{v}\right\|^{2}-\left(\varepsilon^{\prime}\right)^{2} \frac{\Theta_{n}^{2}\left(\boldsymbol{a}_{n}\right)}{\left\|\Theta_{n}^{\prime}\left(\boldsymbol{a}_{n}\right)\right\|^{2}},
\end{aligned}
$$


where we used the property of Thm. 24, and the definition of the subgradient $\Theta_{n}^{\prime}\left(\boldsymbol{a}_{n}\right)$. As a result, $\left\|\boldsymbol{a}_{n+1}-\boldsymbol{v}\right\| \leq\left\|\boldsymbol{a}_{n}-\boldsymbol{v}\right\|$. Notice, that this holds true also for the case where $\Theta_{n}^{\prime}\left(\boldsymbol{a}_{n}\right)=0$. Now, if we apply $\inf _{\boldsymbol{v} \in \Omega_{n}}$ on both sides of the previous inequality, then we establish the claim of Thm. 111.

2. Fix $n \in \overline{n_{0}, n_{0}+N-1}$. Assume that $\Theta_{n}^{\prime}\left(\boldsymbol{a}_{n}\right) \neq 0$. Then, notice by the convexity of the function $\|\cdot\|^{2}$ that

$$
\begin{aligned}
\frac{\Theta_{n}^{2}\left(\boldsymbol{a}_{n}\right)}{\left\|\Theta_{n}^{\prime}\left(\boldsymbol{a}_{n}\right)\right\|^{2}} & =\frac{\sum_{i \in \mathcal{I}_{n}} \omega_{i}^{(n)} d^{2}\left(\boldsymbol{a}_{n}, S_{i}\left[\epsilon_{i}\right]\right) \sum_{i \in \mathcal{I}_{n}} \omega_{i}^{(n)} d^{2}\left(\boldsymbol{a}_{n}, S_{i}\left[\epsilon_{i}\right]\right)}{\left\|\sum_{i \in \mathcal{I}_{n}} \omega_{i}^{(n)}\left(\boldsymbol{a}_{n}-P_{S_{i}\left[\epsilon_{i}\right]}\left(\boldsymbol{a}_{n}\right)\right)\right\|^{2}} \\
& \geq \frac{1}{q} \sum_{i \in \mathcal{I}_{n}} d^{2}\left(\boldsymbol{a}_{n}, S_{i}\left[\epsilon_{i}\right]\right) \\
& \geq \frac{1}{q} \max \left\{d^{2}\left(\boldsymbol{a}_{n}, S_{j}\left[\epsilon_{j}\right]\right): j \in \mathcal{J}_{n}\right\} .
\end{aligned}
$$

Hence, by (21),

$$
\begin{aligned}
\left\|\boldsymbol{a}_{n+1}-\boldsymbol{v}\right\|^{2} \leq & \left\|\boldsymbol{a}_{n}-\boldsymbol{v}\right\|^{2} \\
& -\frac{\left(\varepsilon^{\prime}\right)^{2}}{q} \max \left\{d^{2}\left(\boldsymbol{a}_{n}, S_{j}\left[\epsilon_{j}\right]\right): j \in \mathcal{J}_{n}\right\} .
\end{aligned}
$$

Notice also that (22) holds true also for the case where $\Theta_{n}^{\prime}\left(\boldsymbol{a}_{n}\right)=0$. If we take the infimum over all $\boldsymbol{v} \in \bigcap_{n=n_{0}}^{n_{0}+N-1} \Omega_{n}$ on both sides of (22), and if we add the resulting inequality for all values of $n \in \overline{n_{0}, n_{0}+N-1}$, then the claim of Thm. 112 is established.

3. (a) Choose arbitrarily any $\boldsymbol{v} \in \Omega$. Then, by definition, there exists an $n_{0}$ such that $\boldsymbol{v} \in \bigcap_{n \geq n_{0}} \Omega_{n}$. Clearly, (22) leads to $\left\|\boldsymbol{a}_{n+1}-\boldsymbol{v}\right\| \leq\left\|\boldsymbol{a}_{n}-\boldsymbol{v}\right\|, \forall n \geq n_{0}$, i.e., $\left(\left\|\boldsymbol{a}_{n}-\boldsymbol{v}\right\|\right)_{n \geq n_{0}}$ is monotonically nonincreasing, and thus convergent. This result implies also that the sequence $\left(\boldsymbol{a}_{n}\right)_{n \in \mathbb{N}}$ is bounded, and that $\mathfrak{C}\left(\left(\boldsymbol{a}_{n}\right)_{n \in \mathbb{N}}\right) \neq \emptyset[29]$.

(b) Let $\boldsymbol{v} \in \Omega$ and $n_{0}$ as previously. We have already seen that $\forall \boldsymbol{v} \in \Omega,\left(\left\|\boldsymbol{a}_{n}-\boldsymbol{v}\right\|^{2}\right)_{n \in \mathbb{N}}$ is convergent. Thus, it is Cauchy, and

$$
\lim _{n \rightarrow \infty}\left(\left\|\boldsymbol{a}_{n}-\boldsymbol{v}\right\|^{2}-\left\|\boldsymbol{a}_{n+1}-\boldsymbol{v}\right\|^{2}\right)=0 .
$$

Now, a simple inspection of (22) and (23) establish the claim of Thm. 13b,

(c) Notice that $\forall n \geq n_{0}, \Theta_{n}^{\prime}\left(\boldsymbol{a}_{n}\right)=\mathbf{0}$ iff $\boldsymbol{a}_{n} \in \operatorname{lev} \leq 0_{\leq}\left(\Theta_{n}\right)=\bigcap_{i \in \mathcal{I}_{n}} S_{i}\left[\epsilon_{i}\right] \neq \emptyset$. Hence, for such $n$, (19) takes the following equivalent form:

$$
\boldsymbol{a}_{n+1}=T_{\mathrm{GT}}^{(K)} T_{\Theta_{n}}^{\left(\lambda_{n}\right)}\left(\boldsymbol{a}_{n}\right),
$$

where the mapping $T_{\Theta_{n}}$ is the subgradient projection mapping with respect to the convex $\Theta_{n}$ defined as [29]: $T_{\Theta_{n}}^{\left(\lambda_{n}\right)}(\boldsymbol{a}):=\boldsymbol{a}-\lambda_{n} \frac{\Theta_{n}(\boldsymbol{a})}{\left\|\Theta_{n}^{\prime}(\boldsymbol{a})\right\|^{2}} \Theta_{n}^{\prime}(\boldsymbol{a})$, if $\boldsymbol{a} \notin \operatorname{lev}_{\leq 0}\left(\Theta_{n}\right)$, and $T_{\Theta_{n}}^{\left(\lambda_{n}\right)}(\boldsymbol{a}):=\boldsymbol{a}$, if $\boldsymbol{a} \in \operatorname{lev}_{\leq 0}\left(\Theta_{n}\right)$. The equations (20) and (23) imply that $\lim _{n \rightarrow \infty} \frac{\Theta_{n}^{2}\left(\boldsymbol{a}_{n}\right)}{\left\|\Theta_{n}^{\prime}\left(\boldsymbol{a}_{n}\right)\right\|^{2}}=0$. It is a matter of simple algebra to show also that $\left\|\left(I-T_{\Theta_{n}}^{\left(\lambda_{n}\right)}\right)\left(\boldsymbol{a}_{n}\right)\right\|=\lambda_{n} \frac{\Theta_{n}\left(\boldsymbol{a}_{n}\right)}{\left\|\Theta_{n}^{\prime}\left(\boldsymbol{a}_{n}\right)\right\|} \leq 2 \frac{\Theta_{n}\left(\boldsymbol{a}_{n}\right)}{\left\|\Theta_{n}^{\prime}\left(\boldsymbol{a}_{n}\right)\right\|}$. As such,

$$
\lim _{n \rightarrow \infty}\left(I-T_{\Theta_{n}}^{\left(\lambda_{n}\right)}\right)\left(\boldsymbol{a}_{n}\right)=\mathbf{0}
$$


A remarkable property of the subgradient projection mapping is the following [29]: $\forall \boldsymbol{a} \in \mathbb{R}^{L}, \forall \boldsymbol{v} \in$ $\operatorname{lev}_{\leq 0}\left(\Theta_{n}\right) \neq \emptyset$

$$
\frac{2-\lambda_{n}}{\lambda_{n}}\left\|\boldsymbol{a}-T_{\Theta_{n}}^{\left(\lambda_{n}\right)}(\boldsymbol{a})\right\|^{2} \leq\|\boldsymbol{a}-\boldsymbol{v}\|^{2}-\left\|T_{\Theta_{n}}^{\left(\lambda_{n}\right)}(\boldsymbol{a})-\boldsymbol{v}\right\|^{2} .
$$

By (24) and Thm. 21, we can see that $J_{\boldsymbol{a}_{n+1}}^{(K)}=J_{T_{\Theta_{n}}^{\left(\lambda_{n}\right)}\left(\boldsymbol{a}_{n}\right)}^{(K)}$. Now, notice by Thm. 244 and (26) that $\forall \boldsymbol{v} \in \Omega$,

$$
\begin{aligned}
\| T_{\Theta_{n}}^{\left(\lambda_{n}\right)}\left(\boldsymbol{a}_{n}\right) & -T_{\mathrm{GT}}^{(K)} T_{\Theta_{n}}^{\left(\lambda_{n}\right)}\left(\boldsymbol{a}_{n}\right) \|^{2} \\
\leq & \left\|T_{\Theta_{n}}^{\left(\lambda_{n}\right)}\left(\boldsymbol{a}_{n}\right)-\boldsymbol{v}\right\|^{2}-\left\|T_{\mathrm{GT}}^{(K)} T_{\Theta_{n}}^{\left(\lambda_{n}\right)}\left(\boldsymbol{a}_{n}\right)-\boldsymbol{v}\right\|^{2} \\
= & \left\|T_{\Theta_{n}}^{\left(\lambda_{n}\right)}\left(\boldsymbol{a}_{n}\right)-\boldsymbol{v}\right\|^{2}-\left\|\boldsymbol{a}_{n+1}-\boldsymbol{v}\right\|^{2} \\
\leq & \left\|\boldsymbol{a}_{n}-\boldsymbol{v}\right\|^{2}-\frac{2-\lambda_{n}}{\lambda_{n}}\left\|\boldsymbol{a}_{n}-T_{\Theta_{n}}^{\left(\lambda_{n}\right)}\left(\boldsymbol{a}_{n}\right)\right\|^{2} \\
& \quad\left\|\boldsymbol{a}_{n+1}-\boldsymbol{v}\right\|^{2} \leq\left\|\boldsymbol{a}_{n}-\boldsymbol{v}\right\|^{2}-\left\|\boldsymbol{a}_{n+1}-\boldsymbol{v}\right\|^{2} .
\end{aligned}
$$

Thus, by (23), we obtain

$$
\lim _{n \rightarrow \infty}\left(I-T_{\mathrm{GT}}^{(K)}\right) T_{\Theta_{n}}^{\left(\lambda_{n}\right)}\left(\boldsymbol{a}_{n}\right)=\mathbf{0} .
$$

By Thm. 113a, choose any $\hat{\boldsymbol{a}}_{*} \in \mathfrak{C}\left(\left(\boldsymbol{a}_{n}\right)_{n \in \mathbb{N}}\right)$. Thus, there exists a subsequence $\left(\boldsymbol{a}_{n_{k}}\right)_{k \in \mathbb{N}}$ such that $\lim _{k \rightarrow \infty} \boldsymbol{a}_{n_{k}}=\hat{\boldsymbol{a}}_{*}$. Hence, by (25) $\lim _{k \rightarrow \infty} T_{\Theta_{n_{k}}}^{\left(\lambda_{n_{k}}\right)}\left(\boldsymbol{a}_{n_{k}}\right)=\hat{\boldsymbol{a}}_{*}$. This result, (28), and Thm. 213 lead to $\hat{\boldsymbol{a}}_{*} \in \operatorname{Fix}\left(T_{\mathrm{GT}}^{(K)}\right)$. Since $\hat{\boldsymbol{a}}_{*}$ was chosen arbitrarily, we obtain the desired $\mathfrak{C}\left(\left(\boldsymbol{a}_{n}\right)_{n \in \mathbb{N}}\right) \subset \operatorname{Fix}\left(T_{\mathrm{GT}}^{(K)}\right)$.

\section{References}

[1] E. Candès, J. Romberg, and T. Tao. Robust uncertainty principles: exact signal reconstruction from highly incomplete frequency information. IEEE Trans. Inform. Theory, 52(2):489-509, Feb. 2006 .

[2] David L. Donoho. Compressed sensing. IEEE Trans. Inform. Theory, 52:1289-1306, 2006.

[3] T. Blumensath and M. E. Davies. Normalized iterative hard thresholding: Guaranteed stability and performance. IEEE Journal of Selected Topics in Signal Processing, 4(2):298-309, Apr. 2010.

[4] T. Blumensath. Accelerated iterative hard threshoding. Signal Processing, 92(3):752-756, Mar. 2012 .

[5] S. Foucart. Hard thresholding pursuit: an algorithm for compressive sensing. SIAM J. Numerical Analysis, 49(6):2543-2563, 2011.

[6] Y. Chen, Y. Gu, and A. O. Hero. Sparse LMS for system identification. In Proceedings of the IEEE ICASSP, pages 3125-3128, 2009.

[7] D. Angelosante, J. A. Bazerque, and G. B. Giannakis. Online adaptive estimation of sparse signals: Where RLS meets the $\ell_{1}$-norm. IEEE Trans. Signal Proc., 58(7):3436-3447, July 2010. 
[8] B. Babadi, N. Kalouptsidis, and V. Tarokh. SPARLS: The sparse RLS algorithm. IEEE Trans. Signal Proc., 58(8):4013-4025, Aug. 2010.

[9] G. Mileounis, B. Babadi, N. Kalouptsidis, and V. Tarokh. An adaptive greedy algorithm with application to nonlinear communications. IEEE Trans. Signal Proc., 58(6):2998-3007, June 2010.

[10] Y. Murakami, M. Yamagishi, M. Yukawa, and I. Yamada. A sparse adaptive filtering using timevarying soft-thresholding techniques. In Proceedings of the IEEE ICASSP, pages 3734-3737, Dallas: USA, Mar. 2010.

[11] Y. Kopsinis, K. Slavakis, and S. Theodoridis. Online sparse system identification and signal reconstruction using projections onto weighted $\ell_{1}$ balls. IEEE Trans. Signal Proc., 59(3):905-930, Mar. 2011.

[12] G. Su, J. Jin, Y. Gu, and J. Wang. Performance analysis of $\ell_{0}$ norm constraint least mean square algorithm. IEEE Transactions on Signal Processing, 60(5):2223-2235, May 2012.

[13] I. E. Frank and J. H. Friedman. A statistical view of some chemometrics regression tools. Technometrics, 35(2):109-135, 1993.

[14] R. Tibshirani. Regression shrinkage and selection via the LASSO. J. Royal. Statist. Soc. B., 58(1):267-288, 1996.

[15] H.-Y. Gao and A. G. Bruce. Waveshrink with firm shrinkage. Statistica Sinica, 7(4):875-892, 1997.

[16] H.-Y. Gao. Wavelet shrinkage denoising using the non-negative garrote. Journal of Computational and Graphical Statistics, 7(4):pp. 469-488, Dec. 1998.

[17] T. Tao and B. Vidakovic. Almost everywhere behavior of general wavelet shrinkage operators. Applied and Computational Harmonic Analysis, 9(1):72-82, 2000.

[18] A. Antoniadis and J. Fan. Regularization of wavelet approximations. J. American Statistical Association, 96:939-967, 2001.

[19] J. Fan and R. Li. Variable selection via nonconcave penalized likelihood and its oracle properties. J. American Statistical Association, 96:1348-1360, 2001.

[20] H. Zou. The adaptive LASSO and its oracle properties. Journal of the American Statistical Association, 101:1418-1429, Dec. 2006.

[21] A. Antoniadis. Wavelet methods in statistics: some recent developments and their applications. Statistics Surveys, 1:16-55, 2007.

[22] H. Zou and R. Li. One-step sparse estimates in nonconcave penalized likelihood models. The Annals of Statistics, 36(4), 2008.

[23] Y. She. Thresholding-based iterative selection procedures for model selection and shrinkage. Electronic Journal of Statistics, 3:384-415, 2009.

[24] C.-H. Zhang. Nearly unbiased variable selection under minimax concave penalty. Annals of Statistics, 38(6):894-942, 2010. 
[25] J. H. Friedman, T. Hastie, and R. Tibshirani. Regularization paths for generalized linear models via coordinate descent. Journal of Statistical Software, 33(1), Feb. 2010.

[26] N. Simon, J. H. Friedman, T. Hastie, and R. Tibshirani. Regularization paths for Cox's proportional hazards model via coordinate descent. Journal of Statistical Software, 39(5):1-15, 2011.

[27] R. Mazumder, J. H. Friedman, and T. Hastie. SPARSENET: Coordinate descent with nonconvex penalties. J. American Statistical Association, 106(495):1125-1138, Sept. 2011.

[28] K. Goebel and W. A. Kirk. Topics in Metric Fixed Point Theory. Cambridge University Press, 1990.

[29] H. H. Bauschke and P. L. Combettes. Convex Analysis and Monotone Operator Theory in Hilbert Spaces. Springer, 2011.

[30] P. L. Combettes. The foundations of set theoretic estimation. Proc. IEEE, 81(2):182-208, 1993.

[31] I. Yamada and N. Ogura. Adaptive projected subgradient method for asymptotic minimization of sequence of nonnegative convex functions. Numerical Functional Analysis and Optimization, 25(7\&8):593-617, 2004.

[32] K. Slavakis, I. Yamada, and N. Ogura. The adaptive projected subgradient method over the fixed point set of strongly attracting nonexpansive mappings. Numerical Functional Analysis and Optimization, 27(7\&8):905-930, 2006.

[33] K. Slavakis and I. Yamada. The adaptive projected subgradient method constrained by families of quasi-nonexpansive mappings and its application to online learning. To appear in the SIAM Journal of Optimization, 2013.

[34] S. Werner and P. Diniz. Set-membership affine projection algorithm. IEEE Signal Processing Letters, 8(8):231-235, Aug. 2001.

[35] O. Hoshuyama, R. A. Goubran, and A. Sugiyama. A generalized proportionate variable step-size algorithm for fast changing acoustic environments. In Proceedings of IEEE ICASSP, volume 4, pages 161-164, 2004.

[36] C. Paleologu, S. Ciochina, and J. Benesty. An efficient proportionate affine projection algorithm for echo cancellation. IEEE Signal Processing Letters, 17(2):165-168, 2010.

[37] R. T. Rockafellar and R. J.-B. Wets. Variational Analysis. Springer, Berlin, 2004.

[38] Y. M. Lu and M. N. Do. A theory for sampling signals from a union of subspaces. IEEE Transactions on Signal Processing, 56(6):2334-2345, June 2008.

[39] Y. C. Eldar and M. Mishali. Robust recovery of signals from a structured union of subspaces. IEEE Trans. Inform. Theory, 55(11):5302-5316, Nov. 2009.

[40] Y. Kopsinis, K. Slavakis, S. Theodoridis, and S. McLaughlin. Generalized thresholding sparsityaware algorithm for low complexity online learning. In Proceedings of IEEE ICASSP, pages 32773280, Kyoto: Japan, Mar. 2012.

[41] S. Haykin. Adaptive Filter Theory. Prentice-Hall, New Jersey, 3rd edition, 1996. 
[42] S. Theodoridis, K. Slavakis, and I. Yamada. Adaptive learning in a world of projections: a unifying framework for linear and nonlinear classification and regression tasks. IEEE Signal Processing Magazine, 28(1):97-123, Jan. 2011.

[43] S. Chouvardas, K. Slavakis, Y. Kopsinis, and S. Theodoridis. A sparsity promoting adaptive algorithm for distributed learning. IEEE Trans. Signal Processing, 60(10):5412-5425, Oct. 2012.

[44] S. Chouvardas, K. Slavakis, S. Theodoridis, and I. Yamada. A stochastic analysis of the hyperslabbased adaptive projected subgradient method. Submitted for publication to the IEEE Signal Processing Letters (manusrcipt ID: SPL-11972-2012).

[45] M. Loève. Probability Theory, volume 1. Springer-Verlag, New York, fourth edition, 1977.

[46] P. L. Combettes and J.-C. Pesquet. Proximal splitting methods in signal processing. In Fixed-Point Algorithms for Inverse Problems in Science and Engineering. Springer-Verlag, 2011.

[47] Y. Kopsinis, K. Slavakis, S. Theodoridis, and S. McLaughlin. Thresholding-based sparsity-aware online algorithms of linear complexity. in preparation.

[48] Y. Kopsinis, K. Slavakis, S. Theodoridis, and S. McLaughlin. Thresholding-based online algorithms of complexity comparable to sparse LMS methods. Submitted to the 2013 IEEE International Symposium on Circuits and Systems (ISCAS), Oct. 2012.

[49] J. Duchi, S. S-Shwartz, Y. Singer, and T. Chandra. Efficient projections onto the $\ell_{1}$-ball for learning in high dimensions. In Proceedings of International Conference on Machine Learning (ICML), pages 272-279, 2008.

[50] I. Daubechies, M. Fornasier, and I. Loris. Accelerated projected gradient method for linear inverse problems with sparsity constraints. Journal of Fourier Analysis and Applications, 14(5-6):764-792, Dec. 2008.

[51] D. A. Lorenz. Convergence rates and source conditions for Tikhonov regularization with sparsity constraints. Journal of Inverse and Ill-posed Problems, 16(5):463-478, 2008. 